\title{
7 Strukturmuster und Diskursfunktionen
}

Zusammenfassung: Im Kapitel wird der Frage nachgegangen, welche prototypischen sprachstrukturellen und diskursfunktionalen Eigenschaften dem erhobenen Inventar (über ihre Orientierungsfunktion hinaus) zuzusprechen sind. Hierfür werden die Konstruktionen des erhobenen Bestands an über 520 MOD-Token nach dominanten Klassenzugehörigkeiten klassifiziert. Den Strukturmustern lassen sich diskursfunktionale Bedingungen, Gegebenheiten sowie spezifische koloniale Akteursgruppen im Deutschen Reich zuordnen.

\subsection{Klassenzugehörigkeiten der MOD-Token}

Durch die Typisierung des Gesamtbestands aller MOD-Token konnten Distributionen im Hinblick auf quantitativ dominante kommemorative Marker aufgezeigt werden. Zur Beantwortung der Leitfrage der vorliegenden Arbeit sind jene Distributionen hinsichtlich ihrer diskursfunktionalen Dimensionen zu analysieren. Um herauszufinden, welche kolonisatorischen Wissenskonzepte mittels kolonial motivierter Straßenbenennungspraktiken im öffentlichen Raum der deutschen Metropole fixiert werden sollten, wurde ein Verfahren gewählt, das strukturelle Muster der über 520 MOD-Token aufzeigt, die in einem zweiten Schritt hinsichtlich ihrer Diskursfunktionen analysiert werden können. Dass mit der inventarbezogenen Kategorisierung der MOD-Konstruktionen nach den frequenten lexikologischen und insbesondere onymischen Klassen Funktionen von Kolonialtoponymen sichtbar gemacht werden können, zeigen Stolz und Warnke (2015: 143-148) in einem frühen Beitrag am Beispiel verschiedener Konstruktionsmuster deanthroponymischer Makrotoponyme in den deutschen Kolonien auf. Im Einleitungsbeitrag des ersten kolonialtoponomastischen Kompendiums zeigen die Herausgeber (Stolz und Warnke 2018b: 13) an der zur Zeit der französischen Kolonialherrschaft in Algerien erfolgten Benennung Philippeville die kommemorativfunktionale Dimension des anthroponymischen Modifikators auf:

\footnotetext{
Dieses Kolonialtoponym [...] gehört zur Klasse der Eponyme, weil für seine Bildung eine andere Namenklasse (hier: Anthroponyme) zurückgegriffen wird [...]. Allein dies verweist schon auf die diskurslinguistisch wichtige Beobachtung, dass im kolonialen Namenprojekt Personalität lokalisiert wird. Dieses Muster begegnet uns immer wieder.
}

Im kolonialen Namenprojekt des kolonisierten Raumes wird damit eine Struktur gewählt, die seit der Neuzeit bereits zur Benennung von Städten (bspw. Ludwigshafen 1843), Stadtteilen (bspw. Julius- und Auguststadt in Wolfenbüttel im 17. Jh.) und Straßen (bspw. Gutenbergplatz in Mainz 1804) und damit auch für อ 0 Open Access. () 2021 Verena Ebert, publiziert von De Gruyter. (cc)BY-ND Dieses Werk ist lizenziert unter der Creative Commons Attribution-NoDerivatives 4.0 International Lizenz. https://doi.org/10.1515/9783110718133-008 
die Makro- und Mikrotoponyme im deutschsprachigen Raum geläufig, aber offensichtlich nicht so frequent war wie etwa in den Kolonien. Auch für kolonial motivierte Benennungspraktiken in der deutschen Metropole führt das Kategorisierungsverfahren der MOD-Token nach den lexikologisch-onymischen Klassen zu Erkenntnissen der damit intendierten Funktionen (vgl. Ebert 2018: 104-109). Um herauszufinden, welche Modifikatoren dabei maßgeblich in die Konstruktionen eingehen, fand eine tokenbezogene Annotation der 525 MOD-Token nach Appellativa und Onymen mit den jeweiligen Unterklassen statt. ${ }^{112}$ Die Klassifizierung nach den onymischen Klassenzugehörigkeiten erfolgte dabei auf Grundlage ihrer quellenspezifischen Einordnung innerhalb zeitgenössischer wissensvermittelnder Texte: Hierfür wurden neben den enzyklopädischen Werken (vgl. Kausch 1903, Schnee 1920) Benennungsmotiven in administrativen Texten und Adressbüchern sowie weitere historische Quellen berücksichtigt.

Die Häufigkeitsverteilung von appellativischen und onymischen Modifikatoren ist dabei absolut eindeutig: 503 MOD-Token des erstellten Inventars weisen Eigennamen auf, deren Bezug als monoreferentiell zu beschreiben ist (vgl. Nübling et al. 2015: 17-20). Dass deonymische Konstruktionsmuster den numerischen dominanten Fall darstellen, ist insofern wenig überraschend, als dass 26 der 27 ortsübergreifend verfügten MOD-Types Onyme (Ausnahme: Askari) darstellen, denen wiederum über 440 (441) deonymische Konstruktionen zuzuschreiben sind (Diagramm 1, Kap. 6.3.1).

\subsubsection{Appellativa}

Deappellativische Konstruktionen spielen für das erhobene Inventar eine geradezu marginale Rolle: Lediglich 20 SN-Token sind eindeutig den folgenden 17 appellativischen MOD-Types zuzuordnen: Archipel, Askari, Damara, Dattel, Herero, Kakao, Kokos, Kolonie, Koralle, Massai, Palme, Perlmutter, Riff, Somali, Suaheli, Taifun, Tropen. ${ }^{113}$ Bis auf den MOD-Type Askari als „Bezeichnung der farbigen, [...] überwiegend aus Eingeborenen des Schutzgebiets bestehenden Soldaten der Schutztruppe [...] in Deutsch-Ostafrika“ (Schnee 1920 I: 89), der mit usuellen Klassifikatoren für vier Städte des Ortskorpus ${ }^{114}$ erhoben werden konn-

112 Die nur für singuläre Einzelorte erhobenen MOD-Types Afrikanisch und Kolonial stellen Adjektive dar; aus diesem Grund bleiben sie in den folgenden Ausführungen unberücksichtigt. 113 Die MOD-Types Damara, Herero, Massai und Suaheli stellen Bezeichnungen von kolonisierten Bevölkerungsgruppen und damit Appellativa dar (vgl. Nübling et al. 2015: 36).

114 D. s.: Essen (Askaristraße), Hamburg (Askaristieg), München (Askaripfad) und Lodz bzw. Litzmannstadt [Łódź] (Askaristraße). 
te, stellen alle weiteren appellativischen MOD-Types singuläre Phänomene dar. Für den kleinen Bestand an deappellativischen Konstruktionen bleibt darüber hinaus festzuhalten, dass sich dieser zahlenmäßig auf eine Stadt des Ortskorpus konzentriert: Die MoD-Types Archipel, Dattel, Herero, Kakao, Kokos, Kolonie, Koralle, Palme, Perlmutter, Riff, Suaheli und Tropen sind als zweigliedrige Konstruktionen nur für den erst 1939 im Zuge des Polenfeldzugs annektierten Ort Lodz bzw. Litzmannstadt [Łódź] festzustellen. In Kap. 6.1 wurde bereits dargelegt, dass die für die Stadt erhobenen 29 Kolonialismen die durchschnittliche Anzahl an kolonialen Straßenbenennungen bis 1945 übertrifft. Dieser Sondertyp betrifft u. a. auch die mit Appellativa besetzten linken Leerstellen der Konstruktionen. Nähere Erläuterungen $\mathrm{zu}$ jener quantitativen Abweichung und den strukturell atypischen Sonderfällen erfolgt in Kap. 8.3.

\subsubsection{Onyme}

Der Hauptbestand deonymischer Konstruktionen wird in den jeweiligen Unterkapiteln hinsichtlich der quantitativ dominanten Namenklasssen der linksköpfigen Modifikatoren dargelegt: Die Annotation der in Dresden und Breslau [Wrocław] verfügten 21 Benennungen führte zum Zwischenbefund, dass die Konstruktionsmuster in beiden Orten in kommemorativer Intention auf Personen und Orte referieren. In Ebert (2018: 107-108) wurde bereits anhand eines Teilbestandes kolonial motivierter Straßennamen dargelegt, dass

mithilfe deanthroponymischer Bildungen [...] im kolonialen Namenprojekt Personalität lokalisiert [wird] [...]. In besonderer Weise spielt [...] zudem die Integration eben dieses kolonisierten Raumes in Übersee eine Rolle, indem Hodo- bzw. Dromonyme mit toponymischen Modifikatoren verfügt wurden.

Auch für den 503 onymische MOD-Token umfassenden Hauptbestand stellen deanthroponymische und detoponymische Konstruktionen mit PersonenN bzw. OrtsN einen geradezu exorbitanten Anteil dar. Von den 26 ortsübergreifend verfügten onymischen MOD-Types (Diagramm 1, Kap. 6.3.1) stellt nur der MODType Iltis kein Anthroponym bzw. Toponym dar. Er ist der onymischen Klasse der SchiffsN zuzuordnen, spielt aber aufgrund der Verfügung entsprechender zweigliedriger Konstruktionen in nur drei Städten eine ebenfalls untergeordnete Rolle. ${ }^{115}$ Die Benennungen verweisen bezeichnungsmotivisch auf das „deutsche Kanonenboot Iltis; es „[...] zeichnete sich aus in dem chinesischen Boxerauf-

115 Iltisstraße in Berlin, Köln und München. 
stand 1900 bei der Einnahme der Takuforts. Im Oktober 1914 wurde es vor dem Falle Tsingtaus versenkt“ (Adressbuch Köln 1937 IV: 343). In den folgenden Ausführungen werden die Teilbestände deanthroponymischer und detoponymischer Konstruktionsmuster hinsichtlich der jeweiligen MOD-Types und deren tokenbezogenen Verteilungen aufgezeigt. Für das Teilinventar detoponymischer Konstruktionen werden darüber hinaus die jeweiligen toponymischen Unterklassen der MOD-Types erläutert. Die sich aus den musterhaften strukturbezogenen Distributionen ergebenden Diskursfunktionen werden anschließend hinsichtlich der dabei versprachlichten kolonialen Wissensordnungen erläutert.

\subsubsection{Anthroponyme}

Die durch Personennamen besetzten Modifikatoren stellen innerhalb des (Gesamt-)Inventars das numerisch dominante Strukturmuster dar: Bei nahezu 310 (309) SN-Token und damit über der Hälfte (etwa 59\%) aller identifizierten kolonialen SN-Token handelt es sich um deanthroponymische Konstruktionsmuster, die in kommemorativer Intention auf Personen referieren. Vergleicht man solche eindeutigen Distributionen im deutschsprachigen Raum mit den jeweiligen Strukturmustern im kolonialen Namenprojekt des kolonisierten Raumes, sind Unterschiede festzustellen: Wenngleich deanthroponymische Konstruktionen auch innerhalb der kolonialen Makro- und Mikrotoponymie vertreten sind, fanden deanthroponymische Konstruktionen in den Kolonien offenbar nicht so häufig Verwendung. ${ }^{116}$ Innerhalb dieses Teilbestandes anthroponymischer MODToken, die als zweigliedrige Konstruktionen in den öffentlichen Raum der deutschen Metropole bis 1945 verfügt wurden, lässt sich ebenfalls in hohem Maße Musterhaftigkeit feststellen: Unter den drei und mehr als drei Mal verfügten MOD-Types (Diagramm 1, Kap. 6.3) sind folgende neun anthroponymische MODTypes auszumachen: Peters, Lüderitz, Wissmann, Nachtigal, Lettow-Vorbeck,

116 Stolz und Warnke (2015: 147) geben für den erstellten kolonialtoponymischen Bestand in den deutschen Kolonien und Schutzgebieten an, dass es sich bei der kolonialen Makrotoponymie in $20 \%$ aller Fälle um Konstruktionen mit Personennamen im Erstglied handelt. Ortspunkt- und raumübergreifende Analysen zur kolonialen Mikrotoponymie der deutschen Kolonialmacht stehen noch aus. Für die in kolonialzeitlichen Quellen erhobenen deutschsprachigen Straßennamen in Tsingtau (vgl. Schulz und Ebert 2016), Daressalam (vgl. Schulz und Ebert 2017) sowie in mehreren Orten in Deutsch-Südwestafrika (vgl. Aleff 2017), DeutschSamoa und Deutsch-Neuguinea (vgl. Schulz und Aleff 2018) ist festzustellen, dass sich die Häufigkeit der Einschreibung deanthroponymischer Mikrotoponyme recht verschiedenartig gestaltet; nach derzeitigem Forschungsstand ist davon auszugehen, dass es sich bei kommemorativen Ehrungen und/oder Würdigungen von Personen nicht um den numerisch überwiegenden Fall handelt. 
Woermann, Leutwein, Rohlfs, Erckert. Wenngleich die Auswahl gering ausfällt, wurden sie in der Verbindung mit gängigen Klassifikatoren 275 Mal in Großund Mittelstädte verfügt. Dass deanthroponymische Konstruktionen für das Gesamtinventar überwiegen, liegt nicht zuletzt auch an der hochfrequenten Verfügung von Straßennamen, die zum Zeitpunkt der Vergabe Carl Peters und Adolf Lüderitz würdigen und/oder ehren sollten: 131 der deanthroponymischen Konstruktionen sind den entsprechenden MOD-Types Peters und Lüderitz zuzusprechen. Folgende Tabelle stellt alle neun ortsübergreifend verfügte MOD-Types in absteigender Reihenfolge mit Teilen der in den enzyklopädischen Kolonialwerken verfassten Einträge zusammen:

Tab. 10: Distributionen anthroponymischer MOD-Types (ortsübergreifend), Einträge in den Koloniallexika.

\begin{tabular}{|c|c|c|}
\hline MOD-Type & MOD-Token & Lemma in Kausch (1903) und/oder Schnee (1920) \\
\hline Peters & 67 & $\begin{array}{l}\text { „Peters, Dr. C., deutscher Gelehrter, Forscher und Kolonialförderer } \\
\text { [...] } 1891 \text { deutscher Kommissar für DOA [d. i. Deutsch-Ostafrika].“ } \\
\text { (Kausch 1903: 131) } \\
\text { „Peters, Carl, Reichskommissar a. D. [d. i. außer Dienst] [...]. Er } \\
\text { gründete [...] } 1884 \text { die ,Gesellschaft für deutsche Kolonisation ' die } \\
\text { ihn noch in demselben Jahre [...] zu Landerwerbungen an die ostaf- } \\
\text { rikanische Küste aussandte. P. [d. i. Peters] ist [...] als der Begründer } \\
\text { des heutigen Ostafrika anzusehen [...].“ (Schnee } 1920 \text { III: 40) }\end{array}$ \\
\hline Lüderitz & 64 & $\begin{array}{l}\text { „Lüderitz, Adolf, deutscher Kaufmann zu Bremen, der } 1883 \text { die } \\
\text { Bucht von Angra Pequena samt der Umgebung [...] kaufte. } \\
\text { (Lüderitzland).“ (Kausch 1903: 129) } \\
\text { „1885 verkaufte L. [d. i. Lüderitz] seine Besitzungen in Südwestaf- } \\
\text { rika [...] an ein Konsortium, das sie später an die Deutsche Koloni- } \\
\text { algesellschaft für Südwestafrika übertrug.“ (Schnee } 1920 \text { II: 465) }\end{array}$ \\
\hline Wissmann & 47 & $\begin{array}{l}\text { „Wissmann, H. von, deutscher Major und Forscher, [...] Reichs- } \\
\text { Kommissar für DOA [d. i. Deutsch-Ostafrika].“ (Kausch 1903: 137) } \\
\text { „Wissmann, Hermann v., [...] Reichskommissar für Deutsch- } \\
\text { Ostafrika [...]. Auch als Gouverneur von Deutsch-Ostafrika } \\
\text { (1895/96) hatte W. beachtenswerte Erfolge aufzuweisen [...].“ } \\
\text { (Schnee } 1920 \text { II: 721) }\end{array}$ \\
\hline Nachtigal & 32 & $\begin{array}{l}\text { „Nachtigal, Dr. G. [...] } 1884 \text { Togo, Kamerun, DSWA [d. i. Deutsch- } \\
\text { Südwestafrika] Besitz ergreifend und deutsche Fahne hissend.“ } \\
\text { (Kausch 1903: 131) } \\
\text { „Nachtigal, Gustav, [...] } 1884 \text { wurde er durch den Kreuzer ,Möve“ } \\
\text { abgeholt, um die Besitzergreifung von Togo, Kamerun und Deutsch- } \\
\text { Südwestafrika vorzunehmen, welcher Aufgabe er sich mit großer } \\
\text { Gewissenhaftigkeit und Energie unterzog.“(Schnee } 1920 \text { II: 612) }\end{array}$ \\
\hline
\end{tabular}




\begin{tabular}{|c|c|c|}
\hline MOD-Type & MOD-Token & Lemma in Kausch (1903) und/oder Schnee (1920) \\
\hline $\begin{array}{l}\text { Lettow- } \\
\text { Vorbeck }\end{array}$ & 30 & {$[\text { kein Eintrag] }]^{117}$} \\
\hline Woermann & 13 & $\begin{array}{l}\text { „Woermann, Adolf, hervorragender Kaufmann, Reeder und } \\
\text { Kolonialpolitiker [...]. } 1874 \text { trat W. [d. i. Woermann] als Teilhaber in } \\
\text { die Firma Carl Woermann ein [...]. Unter seiner Leitung gelangte } \\
\text { die Firma zu hoher Blüte; } 1884 \text { erfolgte die Abzweigung des } \\
\text { Reedereigeschäftes [...] die heutige Woermann-Linie [...]. Die } \\
\text { Erwerbung der Kolonie Kamerun durch das Deutsche Reich ist } \\
\text { großenteils auf die Verdienste W.s zurückzuführen.“ (Schnee } \\
\text { 1920 III: 724) }\end{array}$ \\
\hline Leutwein & 11 & $\begin{array}{l}\text { „Leutwein, deutscher Major, der bei Naukluft in DSWA [d. i. } \\
\text { Deutsch-Südwestafrika] 1894 die aufständischen Hottentotten } \\
\text { schlug.“ (Kausch 1903: 129) } \\
\text { "Leutwein, Theodor Gotthilf, Ksl. Generalmajor z.D. und früher } \\
\text { Gouverneur von Deutsch-Südwestafrika.“ (Schnee } 1920 \text { II: 452) }\end{array}$ \\
\hline Rohlfs & 8 & $\begin{array}{l}\text { „Rohlfs, Gerhardt, Gelehrter und Forscher, welcher [...] Reisen in } \\
\text { Afrika ausgeführt hat.“ (Kausch 1903: 133) } \\
\text { „Rohlfs, Gerhard, [...] 1880/81 war R. Gesandter in Abessinien und } \\
\text { 1884/85 Generalkonsul in Sansibar. R. [d. i. Rohlfs] steht in der } \\
\text { Reihe der bedeutendsten Afrikaforscher.“ (Schnee } 1920 \text { III: 181) }\end{array}$ \\
\hline Erckert & 3 & $\begin{array}{l}\text { „Erckert, Friedrich von, Hauptmann in der Ksl. [d. i. Kaiserlichen] } \\
\text { Schutztruppe für Südwestafrika, gefallen am 16. März } 1908 \text { im } \\
\text { Gefecht bei Seatsub gegen die Simon - Copper - Hottentotten. [...] } \\
\text { als Abteilungsführer nahm er an den Kämpfen gegen die } \\
\text { Hottentotten [...] hervorragenden Anteil.“ (Schnee } 1920 \text { I: 570-571) }\end{array}$ \\
\hline
\end{tabular}

Für einzelne Benennungen mit dem MOD-Type Woermann sind polyreferenzielle Intentionen zuzuordnen. Die aus den zeitgenössischen Benennungsmotiviken der Einwohnerbücher erhobenen Intentionen zeigen auf, welche Zuschreibungen für die städtische Öffentlichkeit vorgenommen wurden:

Woermannstraße: Adolf Woermann, Großkaufmann und Kolonialpolitiker [...] Mitinhaber des Handelshauses Carl Woermann, gründete 1880 die Deutsch-Westafrikanische Dampferlinie, erwarb 1884 in Verbindung mit Janßen und Thormählen das Kamerungebiet, das seit 1920 Mandatsgebiet des Völkerbundes ist. (Adressbuch Düsseldorf 1940 III: 492)

Woermannstraße: Zum Andenken an die Kolonial-Schiffs-Linie. (Adressbuch Kassel 1939 III: 296)

117 Die Fertigstellung der Lexika erfolgte bereits 1903 bzw. 1914. Das kolonialpolitische Wirken Lettow-Vorbecks ist dagegen erst für die Endphase der de facto-Kolonialzeit einzuordnen (Kap 4). 
Woermannweg: Mitbegründer der Woermann-Linie, welche den deutschen Ostafrikadienst betreibt; erwarb 1884 das Mündungsgebiet des Kamerunflusses als deutschen Kolonialbesitz. (Adressbuch Münster 1941/42 II: 258)

Die Texte beziehen sich primär auf die Person A. Woermanns, zeigen darüber hinaus aber auch Polyreferenzen auf, die sich auf metonymische Übertragungen des Nachnamens für das Handelshaus und die Schiffslinie beziehen. Jene sind ebenfalls in der Enzyklopädie durch zwei weitere Lemmata aufgelistet:

Woermann, C. Hamburg. Betreibt Handel jeder Art. Handelsniederlassungen in Kamerun (unter der Firma Woermann \& Co.) [...]. (Schnee 1920: 724)

Woermann-Linie A.-G., Hamburg, expediert [...] auf 13 verschiedenen Linien regelmäßig monatlich 14 Dampfer von Hamburg nach der Westküste Afrikas [...]. (Schnee 1920: 724)

Die jeweiligen singulären Benennungen wurden daher mit Hinweisen hinsichtlich jener Polyreferenzen, die sich tokenbezogen mit dem MoD-Type Woermann ergaben, vermerkt.

\subsubsection{Toponyme}

Zahlenmäßig an zweiter Stelle stehen detoponymische Konstruktionsmuster: Für über 190 (192) SN-Token und damit für mehr als ein Drittel (etwa 36\%) des erhobenen (Gesamt-)Inventars sind toponymische Modifikatoren festzustellen. Das koloniale Namenprojekt in der deutschen Metropole zeichnet sich damit auch maßgeblich durch die Lokalisation des kolonisierten Raumes in Übersee aus. Innerhalb des Teilbestandes jener toponymischen MOD-Token sind sechzehn MODTypes herauszustellen, die ortsübergreifend erhoben werden konnten und in absteigender Reihenfolge aufgelistet werden: Togo, Windhuk, Kamerun, Tanga, Samoa, Swakopmund, Waterberg, Duala, Otavi, (Neu-)guinea, Tsingtau, Daressalam, Taku(-Fort), (Deutsch-)Südwest(-Afrika), Sansibar, (Deutsch-)Ostafrika. Die Auswahl an frequenten toponymischen Modifikator-Konstruktionen ist damit erheblich vielseitiger als die ortsübergreifend verfügten anthroponymischen MODTypes. Innerhalb des Teilbestands an detoponymischen Konstruktionen sind die drei MOD-Types Togo, Windhuk und Kamerun mit jeweils über 20 SN-Token herauszustellen. Die für deanthroponymische Konstruktionen dargelegte zahlenmäßige Konzentration auf zwei MOD-Types (Peters, Lüderitz) kann für die toponymischen Modifikatoren nicht nachgewiesen werden. Für das auf kolonisierte Orte referierende Teilinventar an detoponymischen Konstruktionen erfolgte eine weitere Annotation der jeweiligen toponymischen Unterklassen der MOD-Token. Dabei ergeben sich folgende Distributionen: 
Tab. 11: Distributionen der Unterklassen toponymischer MOD-Types.

\begin{tabular}{lllll}
\hline $\begin{array}{l}\text { Top. Klassen- } \\
\text { zugehörigkeiten }\end{array}$ & Choronyme & Oikonyme & $\begin{array}{l}\text { Praxonymische } \\
\text { Anteile }\end{array}$ & $\begin{array}{l}\text { Hydro- und } \\
\text { Oronyme }\end{array}$ \\
\hline Anzahl MOD-Token & 89 & 63 & 30 & 10 \\
Distributionen & ca. $46 \%$ & ca. $33 \%$ & ca. $16 \%$ & ca. $5 \%$ \\
\hline
\end{tabular}

Im Ergebnis sind die bereits für Dresden und Breslau [Wrocław] aufgezeigten makrotoponymischen Namenklassen (Kap. 5. 6) auch für das ortsübergreifende Teilinventar aller detoponymischen Konstruktionen dominant: Über 150 der toponymischen erstgliedrigen Modifikator-Konstruktionen stellen Choronyme und Oikonyme dar. Daneben sind 30 toponymische MOD-Token mit praxonymischen Anteilen festzustellen. Alle ortsübergreifend verfügten MOD-Types jener drei toponymischen Unterklassen, die drei und mehr als drei Mal als zweigliedrige Konstruktionen erhoben wurden, werden in Einzelkapiteln mit den jeweiligen tokenbezogenen Distributionen und mit Teilen der in den enzyklopädischen Werken (vgl. Schnee 1920 und/oder Kausch 1903) verfassten Einträge tabellarisch zusammengestellt. Dehydro- und oronymische Konstruktionen stellen singuläre Phänomene dar; aus diesem Grund tauchen sie auch nicht in Diagramm 1 (Kap. 6.3.1) auf. Jene nur für Einzelorte festzustellenden ModifikatorKonstruktionen werden anschließend dargelegt.

\section{a. Choronyme}

Dechoronymische Konstruktionen beziehen sich auf kolonisierte Gebiete in Übersee. Dabei sind sieben MOD-Types herauszustellen, die in der Verbindung usueller Klassifikatoren drei und mehr als drei Mal verfügt wurden. Sie werden in der Tabelle mit den jeweiligen tokenbezogenen Distributionen und mit Einträgen der Koloniallexika aufgeführt:

Tab. 12: Distributionen choronymischer MOD-Types (ortsübergreifend), Einträge in den Koloniallexika.

\begin{tabular}{lll}
\hline MOD-Type & MOD-Token & Lemma in Kausch (1903) und/oder Schnee (1920) \\
\hline Togo & 25 & „Togoland (WA [d. i. Westafrika]), deutsche Kolonie, 1. Mai 1885.“ \\
& (Kausch 1903: 108) \\
& „Das Schutzgebiet T. [d. i. Togo] liegt an der Westküste Afrikas und \\
& zwar an dem “Sklavenküste” genannten Teil des Golfs von Guinea. \\
& Es ist nach der kleinen an der Südostecke des T.sees gelegenen \\
& Landschaft T. benannt, deren Häuptling der erste war, welcher mit \\
& dem deutschen Kommissar Dr. Nachtigal [...] einen Schutzvertrag \\
& abschloß.“ (Schnee 1920 III: 497) \\
\hline
\end{tabular}




\begin{tabular}{|c|c|c|}
\hline MOD-Type & MOD-Token & Lemma in Kausch (1903) und/oder Schnee (1920) \\
\hline amerun & 24 & $\begin{array}{l}\text { „(deutsche Kolonie in WA [d. i. Westafrika]) [...] Regierungs- und } \\
\text { Missionsstat., deutsche Telegraphenanstalt 21. Februar 1893, } \\
\text { Kabel über Bonny seit diesem Tage mit Europa verbunden, deut- } \\
\text { sche Postanstalt, die allerdings jetzt (1902) nach Duala verlegt } \\
\text { worden ist [...].“ (Kausch 1903: 49) } \\
\text { „Die Kolonie K. [d. i. Kamerun] liegt im innersten Winkel der Bucht } \\
\text { von Guinea, bildet also einen Teil der Guineaküste und trennt das } \\
\text { sog. Ober-Guinea von Nieder-Guinea.“ (Schnee } 1920 \text { II: 169) }\end{array}$ \\
\hline Samoa & 12 & $\begin{array}{l}\text { „Samoainseln = Schifferinseln, Navigatoren (DM [d. i. Deutsch-Mikro- } \\
\text { nesien]) [...] Deutscher Besitz: Upolu, Sawaii.“ (Kausch 1903: 98) } \\
\text { „S. [d. i. Samoa] liegt mitten im Pazifischen Ozean [...]. An der Spitze } \\
\text { der gesamten Verwaltung steht ein vom Kaiser ernannter Gouver- } \\
\text { neur. Seit der Übernahme der deutschen Schutzherrschaft über die } \\
\text { S.inseln, d. h. seit dem 1. März 1900, bekleidete dieses Amt der [...] } \\
\text { jetzige Staatssekretär Dr. Solf. [...] Der Sitz des Gouverneurs ist Apia } \\
\text { auf der Insel Upolu.“ (Schnee } 1920 \text { III: 214, 236) }\end{array}$ \\
\hline $\begin{array}{l}\text { (Neu-) } \\
\text { Guinea }\end{array}$ & 5 & $\begin{array}{l}\text { „Deutsch-Neu-Guinea (KWL [d. i. Kaiser Wilhelmsland]) und BA [d. } \\
\text { i. Bismarck-Archipel] [...].“ (Kausch 1903: 30) } \\
\text { „Neuguinea, die größte Insel der Südsee [...]. Im Jahre } 1828 \text { nahmen } \\
\text { die Niederländer den westlichen Teil der Insel [...] in Beschlag. Den } \\
\text { Rest teilten sich die Engländer und Deutschen durch Vertrag vom } 29 . \\
\text { April } 1886 \text { [...]. Der deutsche Anteil, Kaiser-Wilhelmsland [...], nimmt } \\
\text { den Nordosten der Insel ein.“ (Schnee } 1920 \text { II: 629) }\end{array}$ \\
\hline $\begin{array}{l}\text { (Deutsch-) } \\
\text { Südwest } \\
\text { (-Afrika) }\end{array}$ & 4 & $\begin{array}{l}\text { „Deutsch-Südwestafrika [...] Ursprünglich wurde in den weitesten } \\
\text { Kreisen der Name Angra Pequena für das ganze Schutzgebiet ge- } \\
\text { braucht. [...] Der Kürze halber wird in volkstümlicher Sprechweise } \\
\text { neuerdings vielfach statt der amtlichen Bezeichnung Deutsch- } \\
\text { Südwest oder einfach Südwest gebraucht [...].“ (Schnee } 1920 \text { I: 410) }\end{array}$ \\
\hline $\begin{array}{l}\text { (Deutsch-) } \\
\text { Ostafrika }\end{array}$ & 3 & $\begin{array}{l}\text { „Deutsch-Ostafrika [...] liegt ganz innerhalb der natürlichen Gren- } \\
\text { zen des geographischen Begriffs Ostafrika [...] Der Araberaufstand } \\
\text { hatte gezeigt, daß die Deutsch-Ostafrikanische Gesellschaft sich } \\
\text { ohne Hilfe des Reichs auf die Dauer nicht würde behaupten kön- } \\
\text { nen. Daher übernahm das Deutsche Reich am 1. Jan. } 1991 \text { die } \\
\text { Verwaltung des Schutzgebiets [...] Sitz des Gouvernements wurde } \\
\text { Daressalam. Sämtliche Hoheitsrechte der Deutsch- Ostafrikani- } \\
\text { schen Gesellschaft gingen allmählich auf das Reich über.“ } \\
\text { (Schnee } 1920 \text { I: 357, 405) }\end{array}$ \\
\hline Sansibar & 3 & $\begin{array}{l}\text { „Sansibar [...], Insel DOA [d. i. Deutsch-Ostafrika] vorgelagert unter } \\
\text { eigenem Sultan mit brit. Schutz.“ (Kausch 1903: 100) } \\
\text { „Die Insel S. [d. i. Sansibar] ist die größte Insel des S.archipels } \\
\text { [...]. Das heutige Sultanat S., das britische Zanzibar Protectorate, } \\
\text { ist nur ein kleiner Teil des einstigen Machtbereichs.“ (Schnee } 1920 \\
\text { III: 252-253) }\end{array}$ \\
\hline
\end{tabular}


Neben den hochfrequenten MOD-Types Togo und Kamerun, die auf die gleichnamigen Kolonien des Deutschen Kaiserreichs referieren, ist auch der MOD-Type Samoa herauszustellen, der durch zwölf zweigliedrige Konstruktionen im (Gesamt-)Inventar vertreten ist.

\section{b. Oikonyme}

Für den Bestand oikonymischer Modifikator-Konstruktionen sind MOD-Types auszumachen, die als zweigliedrige Konstruktionen ortsübergreifend Ortskorpus verfügt wurden.

Tab. 13: Distributionen oikonymischer MOD-Types (ortsübergreifend), Einträge in den Koloniallexika.

\begin{tabular}{|c|c|c|}
\hline MOD-Type & MOD-Token & Lemma in Kausch (1903) und/oder Schnee (1920) \\
\hline Windhuk & 25 & $\begin{array}{l}\text { „[...] (DSWA [d. i. Deutsch-Süd-West-Afrika]), O. [d. i. Ort], } \\
\text { Damaraland, deutsches Postamt, Militär- und Missionstat., Reg.- } \\
\text { Sitz, Bezirkshauptmannschaft [...].“ (Kausch 1903: 118) } \\
\text { „Hauptort des Schutzgebietes Deutsch-Südwestafrika, [...] Sitz } \\
\text { der Regierung. W. [d. i. Windhuk] ist sowohl Sitz des Gouverneurs } \\
\text { wie auch der Post- und Zollbehörde [...]. Es ist ferner der Sitz des } \\
\text { Kommandos der Schutztruppe, gleichzeitig auch Garnison einer } \\
\text { größeren Abteilung. Die Rheinische und die Katholische Mission } \\
\text { besitzen Stationen in der Hauptstadt.“ (Schnee } 1920 \text { III: 714) }\end{array}$ \\
\hline Swakopmund & 10 & $\begin{array}{l}\text { „[...] (DSWA), O, Reg.-, Missions- und Militärstation, Bahnstation, } \\
\text { deutsche Postanstalt 30. Mai 1895, Küste des Atl. Ocean, Eisen- } \\
\text { bahn im Bau Swakopmund-Windhoek (fertig } 1902 \text { bis Kapen- } \\
\text { oussen), Postlinie Swakopmund-Walfischbai.“ (Kausch 1903: 105) } \\
\text { „Swakopmund [...], der wichtigste Küstenplatz von Deutsch- } \\
\text { Südwestafrika [...] daß bereits } 1901 \text { in S. [d. i. Swakopmund] etwa } \\
\text { die gleiche Zahl von Einzelfirmen tätig war wie in Windhuk selbst. } \\
\text { Ist auch die Bedeutung von Lüderitzbucht seither ganz erheblich } \\
\text { gestiegen, so hat S. gleichwohl alle Aussicht, dauernd den Rang } \\
\text { des wichtigsten Tores für den Handel des Schutzgebiets zu be- } \\
\text { haupten.“(Schnee } 1920 \text { III: 441) }\end{array}$ \\
\hline Duala & 6 & $\begin{array}{l}\text { „[1] „Duala, Dualla, Volk i. d. Kamerunorten. [2] Duala (K) (d. i. } \\
\text { Kamerun), O, neuer Regierungssitz (früh. Kamerun), deutsches } \\
\text { Postamt [...].“ (Kausch 1903: 32) } \\
\text { „1. Der Ort D. [d. i. Duala] ist der Hauptort der Kolonie Kamerun. } \\
\text { [...] 2. Der Bezirk D. erstreckt sich zwischen den Flüssen Muno und } \\
\text { Dibamba bis zur ersten Hochlandstufe [...]. 3. Die D. sind ein } \\
\text { Bantustamm, der seine Wohnsitze am Kamerunästuar, zwischen } \\
\text { den Flüssen Wuri und Dibamba hat.“(Schnee } 1920 \text { I: 477-479) }\end{array}$ \\
\hline
\end{tabular}




\begin{tabular}{|c|c|c|}
\hline MOD-Type & MOD-Token & Lemma in Kausch (1903) und/oder Schnee (1920) \\
\hline Otavi & 6 & $\begin{array}{l}\text { „(Otavi Minen) [DSWA], O., deutsche Post seit 18. Oktober 1899, } \\
\text { Militärstation, Bergdamaraland, Postlinie: Okahandja-Otavi- } \\
\text { Grootfontein.“ (Kausch 1903: 89) } \\
\text { „Otavi, ein durch seine Kupfererzlager berühmter Platz im äußers- } \\
\text { ten Norden des Hererolandes (Deutsch-Südwestafrika). Er liegt an } \\
\text { der nach ihm benannten Eisenbahn, [...] um in nordöstlicher } \\
\text { Richtung das Minengebiet zu erreichen. [...] Großen Umfang } \\
\text { begann der bergmännische Betrieb [...] mit der } 1906 \text { erfolgten } \\
\text { Fertigstellung der Bahn [...] im Folgejahre hob sich die Ausfuhr } \\
\text { von Erzen zu bedeutender Höhe. Seitdem ist das O. gebiet eine } \\
\text { der für die Entwicklung des Schutzgebiets wichtigsten Landschaf- } \\
\text { ten geworden.“ (Schnee } 1920 \text { II: 691-692) }\end{array}$ \\
\hline Tsingtau & 5 & $\begin{array}{l}\text { „Früher Tsingtaufort DCh [d. i. Deutsch-China], deutsche Post } 26 . \\
\text { Januar 1898, Fernsprecheinrichtung 1. Juni 1899. Bahnprojekte: } \\
\text { Tsintau-Wëishiën-Tsinanfu, } 350 \text { km, Tsintau-Itschoufu, } 190 \text { km } \\
\text { und Verbindung Tsinanfu-Itschoufu } 400 \text { km.“ (Kausch 1903: 109) } \\
\text { „Tsingtau s. Kiautschou.“ (Schnee } 1920 \text { III: 555) „[...] war Tsing- } \\
\text { tau, die Hafenstadt des Schutzgebiets, von vornherein gedacht } \\
\text { als ein Stapelplatz und Umschlaghafen für die seewärts einge- } \\
\text { henden europäischen Waren zur Versorgung des ausgedehnten } \\
\text { chinesischen Hinterlandes einerseits, sowie als Ausfuhr- und } \\
\text { Verteilungshafen für die mannigfachen Erzeugnisse Schantungs } \\
\text { und der anschließenden Provinzen andererseits. [...] Hauptstadt } \\
\text { des Schutzgebiets [.....“ (Schnee } 1920 \text { II: 261-262) }\end{array}$ \\
\hline Daressalam & 4 & $\begin{array}{l}\text { „(DOA [d. i. Deutsch-Ost-Afrika], O, } 20000 \text { Bew., [...] Missionsstat., } \\
\text { deutsches Postamt 4. Oktober 1890 [...] Postlinien: Dar-es-Salaam - } \\
\text { Tabora - Muanza - Bukoba [...].“(Kausch 1903: 29) } \\
\text { „Hauptstadt [...] von Deutsch-Ostafrika, ferner apostolisches } \\
\text { Vikariat der katholischen Mission. [...] Die Stadt D. [d. i. Daressa- } \\
\text { lam] ist unter allen guten Hafenorten der ostafrikanischen Küste } \\
\text { derjenige, der Sansibar, dem ehemaligen Schlüssel des tropi- } \\
\text { schen Ostafrika, am nächsten liegt.“ (Schnee 1920 I: 285) }\end{array}$ \\
\hline
\end{tabular}

Neben dem hochfrequenten MOD-Type Windhuk ist für den Bestand solcher deoikonymischer Konstruktionen der MOD-Type Swakopmund herauszustellen, der zehn Mal Verwendung fand.

Wenngleich in den zentralen enzyklopädischen Werken aus der faktischen Kolonialepoche polyreferenzielle Bezüge für Duala festzustellen sind, ist bei den ortsübergreifend verfügten Konstruktionen mit dem MOD-Type Duala davon auszugehen, dass sie sich vorrangig auf den Hauptort der deutschen Kolonie Kamerun beziehen sollten. Eine tokenbezogene Rekonstruktion zeitgenössischer Benennungsmotiviken aus historischen Straßenverzeichnissen kann nur 
für zwei der sechs SN-Token vorgenommen werden. Für die in nationalsozialistischer Zeit in Bremen und Magdeburg verfügten Konstruktionen sind dabei folgende sich voneinander unterscheidende Motiviken festzustellen:

Dualaweg: Duala, ein Negerstamm in Kamerun. (Adressbuch Bremen 1939 III: 57)

Dualastraße: Duala, Hauptstadt von Kamerun, zur Erinnerung an die verlorenen Kolonien. (Adressbuch München 1941 IV: 174)

Allerdings ist in zeitgenössischen Quellen von der „grossen Unzuverlässigkeit der Dualastämme“ (Puttkamer 1912: $44^{118}$ ) die Rede. Es erscheint daher äußerst fraglich, inwieweit man im öffentlichen Raum der Stadt Bremen tatsächlich die Volksgruppe der Duala mit kolonial motivierten Straßenbenennungen ehren und/oder würdigen wollte. Gewisse Unsicherheiten können bei einer solchen sprachhistorischen Untersuchung nicht völlig lückenlos geklärt werden. Das Archivgut zur Benennung des SN-Tokens Dualaweg in Bremen konnte nicht ermittelt werden. Die im Bremer Straßenverzeichnis von 1939 aufgeführte Motivik ist daher kritisch zu hinterfragen, und zwar auch deshalb, weil alle weiteren innerhalb des Kolonialclusters in Bremen-Oslebshausen verfügten SN-Token auf Orte in den kolonisierten Gebieten referieren. Wahrscheinlicher ist, dass mit der Namenverfügung eine Ehrung und/oder Würdigung des Hauptortes der Kolonie Kamerun verfolgt wurde.

\section{c. Toponymische MOD-Types mit praxonymischen Anteilen}

Innerhalb des Teilbestandes detoponymischer Konstruktionen sind die fünf MOD-Types Tanga, Waterberg, Taku(-Fort), Groß-Nabas und Peking zu verorten, die ausdrucksseitig auf Orte referieren. Gleichzeitig stellen die jeweiligen Modifikator-Konstruktionen „Eigennamen zur Bezeichnung historischer Ereignisse“ (Spitzmüller und Warnke 2011: 141) dar, d. h. für die jeweiligen Konstruktionen sind praxonymische Verwendungsweisen festzustellen:

Praxonyme (<griech. Praxis, 'Tat, Handlung'). Damit sind EN für Ereignisse gemeint [...] also z. B. militärische, politische, wirtschaftliche, kulturelle, gesellschaftliche, sportliche Begebenheiten [Fettdruck im Original] [...]. (Nübling et al. 2015: 317)

Solche praxonymischen Anteile können sowohl in Einträgen zeitgenössischer Koloniallexika der Modifikator-Konstruktionen als auch innerhalb von zeitgenössischen Benennungsmotiviken der SN-Token nachgewiesen werden:

118 Teil der DSDK. 
Groß-Nabas [Fettdruck im Original], Platz in Deutsch-Südwestafrika, an dem am 2. bis 4. Jan. 1905 ein verlustreiches Gefecht der deutschen Truppen gegen die Hottentotten stattfand (s. Hereroaufstand). (Schnee 1920 I: 760)

Groß-Nabas-Straße. Vom 2.-4.1.1905 fand bei Groß-Nabas in Deutsch-Südwestafrika ein Gefecht gegen den Großkapitän der Witboi-Hottentotten statt, an dem die Bayernkompanie hervorragend beteiligt war. (Adressbuch München 1941 IV: 313)

Pekingplatz. Zum Gedächtnis des chinesischen Feldzuges. (Adressbuch Berlin 1908 III: 615)

Bis auf die zwei einzelortsbezogenen Modifikatoren Groß-Nabas und Peking konnten für alle weiteren drei MOD-Types ortsübergreifend verfügte Konstruktionen erhoben werden. Sie werden in der Tabelle mit der jeweiligen Anzahl an entsprechenden SN-Token und den Einträgen in Schnee (1920) dargelegt.

Tab. 14: Distributionen toponymischer MOD-Types mit praxonymischen Anteilen (ortsübergreifend), Einträge in den Koloniallexika.

\section{MOD-Type MOD-Token Lemma bzw. Auszüge aus Beschreibungsteilen anderer Lemmata in Schnee (1920)}

\begin{tabular}{ll}
\hline Tanga $15 \quad \begin{array}{l}\text { „Tanga [...], die nördlichste Hafenstadt von Deutsch-Ostafrika am } \\
\text { Indischen Ozean und die zweitwichtigste des Schutzgebietes.“ } \\
\text { (Schnee III: 454) }\end{array}$
\end{tabular}

Waterberg $10 \quad$ „In W. [d. i. Waterberg] befand sich der Sitz des Hererohäuptlings Kamasembi [...]. Nachdem Kamasembi 1903 gestorben war, erhoben sich [...] die am W. ansässigen Herero [...] im Januar 1904 gegen die Deutschen. Im August desselben Jahres fanden dann die entscheidenden Kämpfe statt, nachdem die Aufrührer auf dem W. von den deutschen Truppen eingeschlossen waren (s. Hereroaufstand).“ (Schnee 1920 III: 691)

„Hereroaufstand. Der Hereroaufstand war der schwerste Eingeborenenaufstand im Schutzgebiet Deutsch-Südwestafrika seit der Besitzergreifung [...]. Erst nach weiterer Verstärkung und Neugliederung der Schutztruppe unter dem Oberbefehl des Generalleutnants v. Trotha [...] wurde der zähe Widerstand der Hereros in dem Entscheidungskampf am Waterberg am 11. Aug. 1904 gebrochen. Ihre Flucht in das Sandfeld, wo sie dem Tode des Verdurstens preisgegeben waren, vollendete ihr Schicksal.“ (Schnee 1920 II: 59)

Taku(-Fort) 4 "Ostasiatische Expedition. Am 22. Juli 1900 wurde der deutsche Gesandte in Peking [...] ermordet. Als Antwort wurde von Deutschland ein Truppen aller Gattungen umfassendes Detachement [...] unter dem Namen ,Ostasiatisches Expeditionskorps“ [...] gebildet. [...] In den letzten Tagen des Juli verließ die Expedition [...] in verschiedenen Staffeln Bremerhaven und traf wohlbehalten Mitte September vor Taku ein. Ungefähr gleichzeitig kam dort der Graf Waldersee, der auf Anregung Rußlands mit dem Oberbefehl über die internationalen Truppen betraut war, an [...].“ (Schnee 1920 II: 689) 
Für zweigliedrige Konstruktionen mit dem MoD-Type Tanga ist neben der Ehrung und/oder Würdigung des Ortes die damit verbundene praxonymische Intention nicht festzustellen, weil das Koloniallexikon im Jahr der dort stattgefundenen Schlacht bereits fertiggestellt wurde (Kap. 4). Die toponymischen und/oder praxonymischen Anteile können allerdings in Benennungsmotiviken jüngerer Namenverfügungen in nationalsozialsozialistischer Zeit nachgewiesen werden. Sie wurden in den folgenden Auszügen fett markiert:

Tangastraße: 1936 nach dem Hafen im ehemaligen Deutsch-Ostafrika. In der Schlacht bei Tanga am 4. November 1914 trug die Schutztruppe einen überwältigenden Sieg über einen weit überlegenen Gegner davon. (Adressbuch Heilbronn 1936 III: 159)

Tangastraße: Tanga, einer der wichtigsten Häfen d. ehem. Deutschostafrikas. Schlacht von Tanga am 4.11.1914. Überwältigender Sieg der kleinen Schutztruppenformationen gegen eine vielfache feindliche Übermacht [...] An der Lüderitzstraße. (Adressbuch Köln 1939 IV: 731)

Tangastraße: Tanga, die nördlichste Hafenstadt des ehemaligen deutschen Schutzgebiets Ostafrika. Hier fand im November 1914 der erste große Kampf der ostafrikanischen Schutztruppe unter Lettow-Vorbeck statt. (Adressbuch München 1941 IV: 621)

Tangastraße: Schlacht bei Tanga 4. Nov. 1914. (Adressbuch Hamburg 1942 IV: 1186)

Die zehn SN-Token mit dem MOD-Type Waterberg beziehen sich auf die Gebirgslandschaft sowie auf den als Poststation ausgebauten und nach dem Gebirge benannten Ort (vgl. Schnee (1920 III: 691). Dass jene Konstruktionen ebenfalls primär praxonymische Bezüge aufweisen, wird neben einer ganzen Schriftenlandschaft ${ }^{119}$ und dem entsprechenden Lemma im Koloniallexikon (Tab. 14) anhand der historischen Benennungsmotiviken entsprechender Namenverfügungen deutlich:

Waterbergstraße: Kämpfe gegen die Herero in Deutsch-Südwestafrika 1904. (Adressbuch Leipzig 1938 II: 492)

Auch das Konstruktionsmuster mit dem MOD-Type Taku(-Fort) weist vorrangig praxonymische Anteile auf. Für den Modifikator liegt kein eigener Eintrag in Schnee (1920) vor, er ist aber im Beschreibungsteil unter dem Lemma Boxeraufstand/Ostasiatische Expedition aufgeführt. Entsprechende Namenverfügungen beziehen sich auf den aus deutscher Sicht erfolgreichen Kampf um die Befestigungsanlagen an der ostasiatischen Küste um die Jahrhundertwende:

119 Man vgl. dazu die über 90 Treffer in der DSDK. 
Takuplatz, Takustraße: Im chinesischen Boxeraufstand 1900 zeichnete die deutsche Marine sich beim Kampf um die Takuforts aus (Iltis- und Lansstraße weisen auch darauf hin). (Adressbuch Köln 1937 IV: 721)

Taku-Fort-Straße: Taku-Fort, bekannt im ostasiatischen Feldzug 1900/01, wurde nach tapferer Verteidigung von den europäischen Truppen erstürmt. (Adressbuch München 1941 IV: 620)

\section{d. Hydro- und Oronyme}

Detoponymische Konstruktionsmuster weisen nur einzelne Benennungen auf, die sich auf Gewässer und Berge in den kolonisierten Räumen beziehen. Für jene singulären Modifikator-Konstruktionen wurden folgende zeitgenössische Zuschreibungen zusammengetragen:

Tab. 15: Singuläre hydronymische und choronymische MOD-Types, Einträge in den Koloniallexika.

\begin{tabular}{|c|c|}
\hline MOD-Type & Lemma in Kausch (1903) und/oder Schnee (1920) \\
\hline Kilimandscharo & $\begin{array}{l}\text { „Kilima-Ndjaro (Nscharo) DOA, der hohe Berg, eigentlich das ganze Gebirge } \\
\text { von der Grösse des deutschen Harzes im Nordosten der Kolonie.“ (Kausch } \\
\text { 1902: 54) } \\
\text { „Der Vulkanberg K. in Deutsch-Ostafrika ist als höchster Berg Afrikas, } \\
\text { ferner wegen seiner landschaftlichen Schönheiten, wegen seiner geogra- } \\
\text { phischen Eigenart eine der am meisten genannten Landschaften der Kolo- } \\
\text { nie. [...] Im Süden erreicht der Rand seine bedeutendste Höhe, die Kaiser- } \\
\text { Wilhelmspitze, die erstmals } 1899 \text { von Hans Meyer (s.d.), dessen Name mit } \\
\text { der Erforschung des K. [d. i. Kilimandscharos] untrennbar verknüpft ist, } \\
\text { erstiegen wurde. Es ist die höchste Stelle Afrikas.“(Schnee } 1920 \text { II: 294) }\end{array}$ \\
\hline Usambara & $\begin{array}{l}\text { „Usambara, Gebirgslandschaft in Deutsch-Ostafrika, der Küste am nächsten } \\
\text { liegender Teil des Ostafrikanischen Randgebirges [...].“ (Schnee } 1920 \text { III: 589) }\end{array}$ \\
\hline Kibo & $\begin{array}{l}\text { „DOA, Höhenpunkt } 5880 \text { m im Kilimanjarogebirge [...] jetzt Kaiser- } \\
\text { Wilhelmspitze benannt.“(Kausch 1903: 54) } \\
\text { „Kibo, Kraterberg, s. Kilimandscharo.“(Schnee } 1920 \text { II: 293) }\end{array}$ \\
\hline Kongo & $\begin{array}{l}\text { „(K. [d. i. Kongo]), Strom, dessen Wassergebiet in den Süden der Kolonie } \\
\text { sich erstreckt [...].“ (Kausch 1903: 58) } \\
\text { „An dem K. [d. i. Kongo], der nächst dem Nil der längste Strom Afrikas ist, } \\
\text { hat Kameruns Südosten Anteil.“ (Schnee } 1920 \text { II: 352) }\end{array}$ \\
\hline Mohasi & $\begin{array}{l}\text { „Mohasi-See DOA, im Norden [...].“ (Kausch 1903: 75) } \\
\text { „Mohasi, einer der Seen des n. Ruanda (Deutsch-Ostafrika), anscheinend ein } \\
\text { Staubecken.“ (Schnee } 1920 \text { II: 586) }\end{array}$ \\
\hline Njassa & $\begin{array}{l}\text { „Njassa [...], einer der großen Seen Ostafrikas, der auf der N- und O- Seite von } \\
\text { deutschem, auf der W-, S- und O-Seite von britischem (Br.- Zentralafrika) und } \\
\text { portugiesischem Gebiet (Prov. Mozambique) umgeben wird (s. Deutsch- } \\
\text { Ostafrika).“(Schnee } 1920 \text { II: 654) }\end{array}$ \\
\hline
\end{tabular}




\begin{tabular}{ll}
\hline MOD-Type & Lemma in Kausch (1903) und/oder Schnee (1920) \\
\hline Sambesi & „DSWA, Fluss im äussersten Nordosten grenzend [...].“ (Kausch 1903: 98). \\
& „Der S., einer der größten Ströme des Weltteils Afrika [...].“ (Schnee 1920 III: \\
& 209) \\
Tanganjika & „Tanganjika, der große See, der auf ungefähr 670 km [...] die Grenze zwischen \\
& Deutsch-Ostafrika und der Kongokolonie bildet.“ (Schnee 1920 III: 455) \\
\hline
\end{tabular}

Die MoD-Types Kongo, Mohasi, Njassa, Sambesi und Tanganjika referieren auf Gewässer (Flüsse oder Seen) in Afrika. Die drei MoD-Types Kilimandscharo, Usambara und Kibo beziehen sich auf Gebirge in den kolonisierten Gebieten. Nur die MOD-Types Kilimandscharo bzw. Usambara sind als koloniale Straßenbenennungen für jeweils zwei Städte ${ }^{120}$ festzustellen. Alle weiteren dehydronymischen und deoronymischen Konstruktionen stellen nur für singuläre Orte ${ }^{121}$ konstatierbare Phänomene dar.

\subsection{Zwischenergebnisse}

Mit der lexikologisch-onymischen Kategorisierung des erhobenen (Gesamt-)Bestands aller MOD-Token konnten die über 520 Kolonialismen hinsichtlich musterhafter Vorkommen kategorisiert werden. Dabei zeigt sich, dass alle ortsübergreifend verfügten MOD-Types (bis auf Lettow-Vorbeck) als eigene Einträge oder im Beschreibungsteil anderer Lemmata in den enzyklopädischen Werken (Kausch 1903, Schnee 1920) aufgeführt sind und somit in einem unmittelbaren zeitgenössischen Zusammenhang mit der deutschen Kolonialepoche verortet wurden. Anhand der quellenbezogenen Zuschreibungen konnten bspw. auch die mit praxonymischen Anteilen verfügten toponymischen MOD-Types im sprachhistorischen Zugriff aufgedeckt und entsprechend eingeordnet werden. Dabei ergaben sich eindeutige Distributionen, die aufzeigen, dass nicht nur die Kolonialtoponymie im kolonisierten Raum, sondern auch das koloniale Namenprojekt in der Metropole „von Systematizität geprägt ist, die es erlaubt, den Gegenstand mit Hilfe von Präferenzregeln adäquat zu beschreiben" (Stolz und Warnke 2018b: 46). Etwa 95\% aller erhobenen Konstruktionen konnten anthroponymischen (59\%) oder toponymischen (36\%) MOD-Token zugeordnet

120 D. s.: Magdeburg (Kilimandscharoweg) und Lodz bzw. Litzmannstadt [Łódź] (Kilimandscharostraße) bzw. Berlin (Usambarastraße) und München (Usambarastraße).

121 D. s.: Kongo-, Sambesi- und Mohasistraße in Berlin, Kibostraße in München, Tanganjikaund Njassastraße in Lodz bzw. Litzmannstadt [Łódź]. 
werden. Systematizitäten konnten auch für die innerhalb der jeweiligen anthroponymischen und toponymischen Teilbestände der verfügten ModifikatorKonstruktionen herausgestellt werden. Auf Grundlage der dargelegten Strukturmuster wird im folgenden Abschnitt auf die diskursorientierte Einordnung der Kolonialismen eingegangen. Dabei soll die Frage beantwortet werden, welche diskursfunktionalen Dimensionen sich aus den jeweils quantitativ dominanten Strukturmustern im Hinblick auf die damit versprachlichten kolonisatorischen Gewissheiten ergeben.

\subsection{Diskursfunktionen}

Mit dem musterhaften Vorkommen an deanthroponymischen und detoponymischen Konstruktionen ist zuallererst festzuhalten, dass kolonial motivierte Namenvergabepraktiken auf eine Ehrung und/oder Würdigung von Personen und von Raum in Übersee abzielen sollten. Übergeordnet ist das Namenprojekt in der deutschen Metropole also damit zu beschreiben, dass eine Fixierung der Parameter Personalität und Lokalität im (Alltags-)Raum der deutschen Gesellschaft angestrebt wurde. Zur Beantwortung der damit versprachlichten genuin kolonisatorischen Gewissheiten sind beide Parameter näher zu untersuchen: Hierfür werden die jeweiligen Teilinventare der deanthroponymischen und detoponymischen Konstruktionsmuster bzw. ihre MOD-Types auf propositionale Einbettungen hinsichtlich ihrer „semantischen Repräsentationen im Rahmen kolonialer Wissensordnungen" (Stolz und Warnke 2018 b: 51) diskutiert. Dies erfolgt durch die Untersuchung der linksköpfigen Konstruktionsmuster im zeitgenössischen transtextuellen Aussagezusammenhang, vorrangig durch Auswertung zeitgenössischer Zuschreibungen aus Einträgen der Koloniallexika, und durch die Auswertung von Benennungsmotiviken aus den historischen Einwohner- bzw. Adressbüchern, die explizit auf die sprachlich-kommunikativen Praktiken der kolonialen Namenvergaben verweisen.

\subsubsection{Personalität}

Dass deanthroponymische Konstruktionen für das zusammengestellte Inventar kolonial motivierter Straßenbenennungen im Deutschen Reich eine quantitativ maßgebliche Rolle einnehmen, ist insofern nicht überraschend, als dass die Würdigung von Personen durch neuzeitliche Straßennamenverfügungen im Deutschen Reich eine usuelle Sprachpraxis darstellt; so können derartige Häufigkeiten administrativ verfügter deanthroponymischer Mikrotoponyme auch in 
einer synchronen Sichtung weiterer, nicht-kolonialer Namenverfügungen bestätigt werden. Schulz und Aleff (2018: 147) zeigen, dass jene Lokalisierung von Personalität für neuzeitliche Straßenbenennungspraktiken im deutschsprachigen Raum eine bedeutende Rolle einnimmt, „und zwar sogar grundsätzlich und damit erheblich über den Bereich der kolonialen Mikrotoponyme hinausgehend. Die Option einer kommemorativen Aufladung durch die Verwendung von Anthroponymen in der Konstruktion von Mikrotoponymen wird nämlich seit dem 19. Jahrhundert systematisch genutzt“. ${ }^{122}$ Aus den rein strukturellen Befunden können damit keine Spezifika ermittelt werden, die symptomatisch für das koloniale Namenprojekt in der Metropole und als typisch koloniale Praxis stehen. Die Beantwortung jener Fragen ist dann möglich, wenn jenes hohe Maß an struktureller Typizität hinsichtlich der damit intendierten Kommemoration der außersprachlichen Referenten untersucht wird. Um herauszufinden, wofür die jeweiligen Personen zum Zeitpunkt der Benennung geehrt und/oder gewürdigt werden sollten, müssen die MOD-Types der ortsübergreifend verfügten deanthroponymischen Konstruktionen sprachhistorisch kontextualisiert werden. Die Untersuchung der mit anthroponymischen MOD-Types versprachlichten kolonisatorischen Wissenskonzepte erfolgt durch die in den Einträgen der Koloniallexika (Tab. 10) vorgenommenen zeitgenössischen Zuschreibungen von Ämtern, Zuständigkeiten $\mathrm{u}$. dgl., aber auch durch die dort getroffenen historischen Aussagen über Tätigkeitsfelder und ausgeübte Handlungen der Personen.

Tab. 16: Historische Zuschreibungen anthroponymischer MOD-Types (ortsübergreifend).

\begin{tabular}{|c|c|}
\hline MOD-Type & Kausch (1903) und/oder Schnee (1920) \\
\hline Peters & $\begin{array}{l}\text { deutscher Kommissar, Kolonialförderer, Reichskommissar, Begründer des heuti- } \\
\text { gen Ostafrika }\end{array}$ \\
\hline Lüderitz & Bucht von Angra Pequena kaufen, Besitzungen in Südwestafrika \\
\hline Wissmann & Reichs-Kommissar, Gouverneur \\
\hline Nachtigal & Besitz ergreifen, deutsche Fahne hissen \\
\hline Woermann & Kaufmann, Kolonialpolitiker, Reedereigeschäft, Erwerbung der Kolonie Kamerun \\
\hline Leutwein & aufständische Hottentotten schlagen, Gouverneur \\
\hline Rohlfs & Reisen in Afrika, Generalkonsul, bedeutendster Afrikaforscher \\
\hline Erckert & Hauptmann der Kaiserlichen Schutztruppe, Kämpfe gegen die Hottentotten \\
\hline
\end{tabular}

122 Der Befund resultiert aus der Sichtung historischer $(1903,1943)$ und gegenwartssprachlicher (2017) Stadtpläne für Frankfurt am Main. 
Sie zeigen, dass mit jenen deanthroponymischen Benennungen allesamt Kolonialakteure des Deutschen Kaiserreichs geehrt und/oder gewürdigt werden sollten, also Personen, die entweder einen entscheidenden Beitrag an der Erwerbung der Kolonien (Adolf Lüderitz, Gustav Nachtigal, Adolf Woermann, Gerhard Rohlfs) trugen, oder während der faktischen Kolonialzeit als Kommissare und/oder Gouverneure hohe administrative Ämter in den deutschen Kolonien $^{123}$ (Carl Peters, Hermann von Wissmann, Theodor Leutwein) inne hatten. Theodor Leutwein und Friedrich von Erckert hatten hohe militärische Ämter bei der Niederschlagung der Kolonialkriege inne, die das Deutsche Kaiserreich gegen die aufständischen Herero und Hottentotten in DeutschSüdwestafrika führte (vgl. Schnee II: 452) .

Ähnliche Zuschreibungen finden sich auch bei den in historischen Adressbüchern dargelegten Benennungsmotiviken für entsprechende Namenverfügungen. Gerade für die in nationalsozialistischer Zeit verfügten deanthroponymischen Namen ist die ortsübergreifende Zuschreibung [Kolonial] pionier[e] frequentiell dominant, die in den folgenden Einträgen fett markiert wurde:

Carl-Peters-Straße: Ben. nach dem Kolonialpionier Dr. Carl Peters. (Adressbuch Braunschweig 1940 III: 39)

Karl-Peters-Straße: Karl Peters [...] Der größte deutsche Kolonialpionier und Gründer der Kolonie Deutsch Ostafrika. (Adressbuch Köln 1937 IV: 578)

Lüderitzstraße: Ben. nach dem Kolonialpionier Lüderitz. (Adressbuch Braunschweig 1940 III: 211)

Lüderitzstraße: 1936 nach Franz Adolf Eduard Lüderitz [...], dem kaufmännischen Pionier des deutschen Kolonialbesitzes und Gründer der ehemaligen deutschen Kolonie DeutschSüdwestafrika. (Adressbuch Heilbronn1936 III: 98)

Lüderitzstraße: Zu Ehren des großen deutschen Kolonialpioniers A. Lüderitz. (Adressbuch Weimar 1937 III: 46)

Nachtigalweg: Zur Erinnerung an den Kolonialpionier Gustav Nachtigal. (Adressbuch Merseburg 1940 II: 204)

123 Man vgl. dazu die jeweiligen Einträge im Koloniallexikon: „Kommissare [Fettdruck im Original] hießen insbesondere diejenigen Beamten, welche vom Kaiser entsandt wurden, um die Verwaltung von Schutzgebieten oder Teilen von solchen zu übernehmen." (Schnee 1920 II: 349) „Gouverneur [Fettdruck im Original]. An der Spitze einer jeden deutschen Kolonie steht gegenwärtig ein G. [d. i. Gouverneur] nachdem die früher für Kolonien von geringerer Bedeutung eingeführten Titel eines Reichskommissars oder Landeshauptmanns fortgefallen sind.“ (Schnee 1920 I: 746) 
Petersstraße: Zur Erinnerung an den Kolonialpionier Carl Peters, Gründer der Kolonie Deutsch-Südwestafrika. (Adressbuch Merseburg 1940 II: 212)

Wissmannstraße: Ben. nach dem Kolonialpionier von Wissmann. (Adressbuch Braunschweig 1940 III: 319)

Wissmannweg: Zur Erinnerung an den Kolonialpionier Hermann Wissmann. (Adressbuch Merseburg 1940 II: 246)

Die nach der faktischen Kolonialzeit erfolgten Benennungen mit dem anthroponymischen MOD-Type Lettow-Vorbeck sollte auf die Person Paul von LettowVorbeck als „Kolonialheld für Kaiser und Führer“ (Schulte-Varendorff 2006) referieren. Dass Paul von Lettow-Vorbeck „schon zu Lebzeiten [...] als heroischer Verteidiger Deutsch-Ostafrikas im Weltkrieg“ (Speitkamp 2004) geehrt und/oder gewürdigt wurde, wird auch durch die adjektivischen Zuschreibungen der Benennungsmotiviken deutlich:

Lettow-Vorbeck-Straße: Paul von Lettow-Vorbeck [...] seit 1918 Kommandeur der Schutztruppe in Deutsch-Ostafrika, das er von 1914-1918 in heldenhafter Weise gegen eine riesige feindliche Übermacht verteidigte. (Adressbuch Köln 1939 IV: 432)

Lettow-Vorbeck-Straße: 1937, benannt nach dem General Lettow-Vorbeck, dem heldenmütigen Verteidiger Deutsch-Ostafrikas im Weltkriege. (Adressbuch Lübeck 1939 III: 550)

Lettow-Vorbeck-Straße: Benannt nach dem heldenhaften Verteidiger Deutsch-Ostafrikas, Generalmajor v. Lettow-Vorbeck. (Adressbuch Wuppertal 1938 III: 253)

Das zahlenmäßig dominante Inventar an ortsübergreifend verfügten deanthroponymischen Benennungen bezog sich in kommemorativer Intention auf Kolonialakteure des Deutschen Kaiserreichs, die aus historischer Sicht einen maßgeblichen Teil an der Aneignung, dem Ausbau und der (militärischen) Sicherung der Herrschaft in den kolonisierten Gebieten hatten und damit ,auf einen vom Namengeber wertgeschätzten Repräsentanten der kolonisierenden Nation [...]" (Stolz und Warnke 2017: 209) verweisen. Dass die Kommemoration auf das Eigene bezogen ist, zeigen auch die in einzelnen metasprachlichen Texten dargelegten Possessivpronomen:

Adolf-Lüderitz-Straße: Zu Ehren von Adolf Lüderitz, der den Ausgangspunkt unserer ehem. Kolonie Deutsch-Südwestafrika erwarb. (Adressbuch Gotha 1941/42 II: 264)

Erckertstraße: Hervorragender Führer in unseren Kolonialkriegen, besonders in den Kämpfen um Südwest-Afrika (Herero-Aufstand 1904). (Adressbuch Hamburg 1940 IV: 354)

Lansstraße: Kapitän Lans, 1900 Befehlshaber unserer Marine im Kampfe um die Takuforts. (Adressbuch Köln 1937 IV: 422) 
Jene kolonisatorische Zentrierung auf das Eigene wird sodann auch durch persönliche Bezüge offensichtlich, die offenbar eine Verbindung rechtfertigen sollten. In diesem Zusammenhang sind gerade die (vorrangig) für Einzelorte erhobenen singulären deanthroponymischen Konstruktionen herauszustellen, die sich auf Kolonialakteure beziehen und gleichzeitig regionale und sogar lokale Bezüge zum Mutterland aufweisen. Auf die regional- bzw. lokalpatriotische Relevanz solcher ortsbezogenen Konstruktionen wird auch in historischen Straßenverzeichnissen eingegangen:

\footnotetext{
Albert-Voigts-Weg: Ben. [d. i benannt] nach dem Braunschweiger Kolonialpionier in Deutsch-Südwest-Afrika. (Adressbuch Braunschweig 1940 III: 39)

Eduard-Haber-Straße: [...] benannt zu Ehren des Tübinger Professors und einstigen Gouverneurs der Kolonie Deutsch-Neuguinea. (Adressbuch Tübingen 1942 III: 19)

Hermann-Blumenau-Straße: Ben. nach dem Braunschweiger Kolonialpionier im Staate Sante Catherine von Brasilien. (Adressbuch Braunschweig 1940 III: 148)

Otto-Finsch-Straße: Ben. nach dem Braunschweiger Vogelforscher und Kolonalpionier in Neu-Guinea. (Adressbuch Braunschweig 1940 III: 239)

Von-Erkert-Platz/Von-Erkert-Straße: [...] Friedrich von Erkert, Hauptmann und erster Kamelreiterführer der Schutztruppe der sog. Bayernkompanie (1869-1908). (Adressbuch München 1941 IV: 668)
}

\subsubsection{Lokalität}

Zur Fixierung kolonisatorischer Gewissheiten in der Metropole steht der Parameter Lokalität durch entsprechende detoponymische Konstruktionsmuster an zweiter Stelle, spielt also neben Personalität die quantitativ zweitwichtigste Rolle. Mit toponymischen Modifikator-Konstruktionen wird eine Integration des kolonisierten Raumes im öffentlichen Raum des Deutschen Reichs vorgenommen. Raumkonzepte sind im gesamten neuzeitlichen Kolonialismus zentral. In diskursfunktionaler Perspektive soll ermittelt werden, inwieweit die aufgezeigten strukturbezogenen Distributionen im Namenprojekt der Metropole selbst Wissensordnungen über kolonisatorisch tradierte Raumkonzepte hervorbringen und welche transtextuellen Zuschreibungen dabei eine übergeordnete Rolle spielten.

\subsubsection{Distributionen und zeitgenössische Zuschreibungen}

Für den Teilbestand detoponymischer Konstruktionsmuster wird eine sprachhistorische Kontextualisierung der jeweiligen ortsübergreifenden choronymi- 
schen, oikonymischen und mit praxonymischen Anteilen festzustellenden toponymischen MOD-Types vorgenommen. Dabei konnten Choronyme und Oikonyme als quantitativ dominante Konstruktionsmuster herausgestellt werden. Die in den enzyklopädischen Werken angelegten Artikel (Tab. 12) zeigen für die Klasse der Choronyme auf, dass diese - mit Ausnahme des MOD-Types Sansibar - auf die Kolonialgebiete des Deutschen Kaiserreichs referieren:

Tab. 17: Historische Zuschreibung choronymischer MOD-Types (ortsübergreifend).

\begin{tabular}{ll}
\hline MOD-Type & Kausch (1903) und/oder Schnee (1920) \\
\hline Togo & deutsche Kolonie, Schutzgebiet, Schutzvertrag abschließen \\
Kamerun & deutsche Kolonie \\
Samoa & deutscher Besitz, Übernahme der deutschen Schutzherrschaft \\
(Neu-)Guinea & Deutsch-Neu-Guinea, größte Insel der Südsee, deutscher Anteil \\
(Deutsch-)Südwest(-Afrika) & deutsches Schutzgebiet \\
(Deutsch-)Ostafrika & Verwaltung des Schutzgebiets durch das Deutsche Reich \\
Sansibar & größte Insel des Sansibararchipels, heutiges Sultanat, britisch \\
\hline
\end{tabular}

Die in den wissensvermittelnden Texten aus der faktischen Kolonialzeit erfolgten Zuschreibungen zeigen für die ortsübergreifend verfügten choronymischen Modifikator-Konstruktionen auf, dass die imperialistische Machtexpansion in Übersee durch den Abschluss von Schutzverträgen auch als deutsche Schutzherrschaft beschrieben wird: Jene Bezeichnung kolonialer Machtkonstellationen und die im Zuge dessen errichteten kolonisierten Räume als Schutzgebiete wird von Warnke und Stolz (2013: 479-483) als Konzept der Reifizierung als entscheidendes Merkmal kolonialer Positionen beschrieben:

Reifizierung im kolonialen Dispositiv meint einerseits die Verdinglichung des dimensionalen Kolonialraums selbst, der als unbelebtes und agensloses Ding erscheint, und es meint eine Verschiebung der Handlungsmacht allein auf die Seite der Kolonisierenden. Damit verbunden ist eine Repräsentation von verfügbaren Kolonialräumen als tatsächlich existierend. (Warnke und Stolz 2013: 480)

Dass geopolitische Machtexpansion und die Errichtung kolonisierter Räume als Schutzgebiete und Schutzherrschaft beschrieben wird, also zum Schutz jener selbst errichteten Länder in Übersee dienen sollten, kann mit den damit „verbundenen Rechtfertigungsabsichten zur kolonialen Unterwerfung“ (Warnke und Stolz 2013: 480) erklärt werden, die mit derartigen Zuschreibungen versprachlicht wurden. Diese sind nicht nur in wissensvermittelnden Texten aus 
der faktischen Kolonialzeit, sondern auch in zeitgenössischen Adressbucheinträgen entsprechender dechoronymischer SN-Token festzustellen, die nach der de facto-Herrschaft in den 1920er und 1930er Jahre von den städtischen Administrationen verfügt wurden: „Togostraße: ehem. deutsches Schutzgebiet in Oberguinea an der Sklavenküste“ (Adressbuch Bremen 1939 III: 283). Dieser Befund ist insofern als Fortsetzung kolonisatorischer Selbstwahrnehmung herauszustellen, als dass bereits Schnee (1920 III: 312) noch in der faktischen Kolonialzeit darauf hinweist, dass die Bezeichnung ein kontrafaktisches Verhältnis zwischen der Metropole und den Kolonien wiedergibt:

\begin{abstract}
Schutzgebiete [Fettdruck im Original] heißen die überseeischen Länder, die unter dem Schutze eines europäischen Staates stehen. [...] Die deutschen Schutzgebiete stehen zu dem Deutschen Reiche in staatsrechtlicher Abhängigkeit; sie sind zwar dem Reichsgebiet nicht einverleibt (Art. 1 Reichsverfassung), gehören aber staatsrechtlich und völkerrechtlich zu dem Reiche. Dieses hat die unbeschränkte Souveränität über sie. Anfangs hat das Reich in den erworbenen Gebieten die Staatsgewalt nicht in vollem Umfange ausgeübt, sondern seine nächste Aufgabe vornehmlich in dem Schutz gesehen. Darauf beruht die Bezeichnung der Erwerbungen als ,Schutzgebiete، und der dem Reiche zustehenden Gewalt als ,Schutzgewalt‘ [...]. Inzwischen hat das Reich auch die weiteren Aufgaben der Staatshoheit: Gesetzgebung, Rechtspflege, Verwaltung übernommen und seine Souveränität nach allen Seiten entfaltet.
\end{abstract}

Anhand der oikonymischen Modifikator-Konstruktionen ist eine geradezu musterhafte „Verschiebung der Handlungsmacht allein auf die Seite der Kolonisierenden“ (Warnke und Stolz 2013: 480) festzustellen. Die inhaltlichen Zuschreibungen in den Koloniallexika (Tab. 13) vermitteln zu solchen Oikonymen kollektiv verfügbare Wissenskonzepte, die man den jeweiligen Orten in den Kolonialgebieten zugeschrieben hat:

Tab. 18: Historische Zuschreibungen oikonymischer MOD-Types (ortsübergreifend).

\begin{tabular}{|c|c|}
\hline MOD-Type & Kausch (1903) und/oder Schnee (1920) \\
\hline Windhuk & $\begin{array}{l}\text { deutsches Postamt, Militär- und Missionsstation, Regierungssitz, Hauptort, } \\
\text { Sitz der Regierung, des Gouverneurs, des Kommandos der Schutztruppe, } \\
\text { Hauptstadt }\end{array}$ \\
\hline Swakopmund & $\begin{array}{l}\text { Regierungs-, Missions- und Militärstation, Bahnstation, deutsche Postanstalt, } \\
\text { wichtigster Küstenplatz, Einzelfirmen, wichtigstes Tor für den Handel }\end{array}$ \\
\hline Duala & neuer Regierungssitz, deutsches Postamt, Hauptort der Kolonie Kamerun \\
\hline Otavi & $\begin{array}{l}\text { Otavi Minen, deutsche Post, Militärstation, Kupfererzlager, Eisenbahn, berg- } \\
\text { männischer Betrieb, Ausfuhr von Erzen, eine der wichtigsten Landschaften }\end{array}$ \\
\hline Tsingtau & $\begin{array}{l}\text { deutsche Post, Bahnprojekte, Hafenstadt, Stapelplatz, Umschlaghafen, Aus- } \\
\text { fuhr- und Verteilungshafen, mannigfache Erzeugnisse, Hauptstadt }\end{array}$ \\
\hline
\end{tabular}




\begin{tabular}{ll}
\hline MOD-Type & Kausch (1903) und/oder Schnee (1920) \\
\hline Daressalam & $\begin{array}{l}\text { Missionsstation, deutsches Postamt, Hauptstadt, guter Hafenort, liegt Sansi- } \\
\text { bar am nächsten }\end{array}$ \\
\hline
\end{tabular}

Wertet man jene Zuschreibungen als (potenziell bekannten) Kontext in Bezug auf Kolonialismus und koloniale Themen im Deutschen Reich, werden die mit derartigen Benennungspraktiken intendierten Diskursfunktionen im Kontext zeitgenössischer kolonialer Raumaneignung und -besetzung evident: Die deoikonymischen Konstruktionsmuster im Raum der deutschen Metropole sollten die in den Kolonien errichteten bzw. ausgebauten administrativen Hauptorte bzw. -städte sowie deren wirtschaftliche und handelsspezifische Zentren ehren und/oder würdigen. Bei den mit derartigen Sprachpraktiken hervorgebrachten Wissenskonzepten ging es primär also darum, auf das eigene kolonialpolitische Handeln und der damit verbundenen Herrschaftssicherung in den Kolonien Bezug zu nehmen: Der vermittelten „Sichtweise des fremden Eindringens in den leeren Raum“, der „vor dem europäischen Zugriff [...] amorph, unstrukturiert, scheinbar unbekannt, unbenannt und unbemannt“" (Speitkamp angenommen) beschrieben wurde, wurde ein aktives Engagement der deutschen Akteure vor Ort (Reisende, Missionare, Militärs, Verwaltungsbeamte, Siedler) entgegengestellt, das die erfolgversprechende Inbesitznahme des kolonisierten Raumes aus Sicht der deutschen Kolonialbefürworter im Kaiserreich legitimieren sollte. Dass gerade mit der Errichtung und dem Ausbau solcher administrativer, wirtschaftlicher und/oder handelsspezifischer Zentren eine Rechtfertigung des kostspieligen kolonialen Projekts intendiert war, verdeutlichen auch zeitgenössische wissensvermittelnde Texte seiner Befürworter:

\footnotetext{
Der weitsichtige Beobachter muss sich sagen, dass kein Menschenalter vergehen dürfte, bis aus dem jetzigen Aufwand an Kraft, Zeit und Geld des Mutterlandes überreicher Gewinn zu ziehen sein wird. [...] Wir richten nicht zwecklos Regierungs- und Stationsbezirke [...] ein, legen nicht umsonst Posten an, bauen nicht ohne Berechtigung Eisenbahn- und Telegraphenlinien und lassen unsere Dampfer nicht unbeladen die Weltmeere durchfahren. Die deutsche Beharrlichkeit, welche im Mutterlande Früchte zeitigt, wird auch in den Kolonien dereinst Segen ernten. (Kausch 1903: 3-4)
}

Mit entsprechenden deoikonymischen Namenvergabepraktiken im öffentlichen Raum der deutschen Metropole, die die (freilich punktuellen) Verwaltungs- und Wirtschaftszentren im kolonisierten Raum ehren und/oder würdigen sollten, konzentrierten sich die administrativen Namengeber auf die aus Sicht der Kolonialbefürworter augenfälligsten Erfolge des neuzeitlichen kolonisatorischen Machstrebens. Das Konzept der Reifizierung wird dabei überdeutlich: Der Fokus 
des kolonialen Projekts lag auf dem eigenen Nutzen, auf der wirtschaftlichen (Aus-)Nutzung der Kolonien, der Rohstoffe und Arbeitskräfte für die Metropole sowie auf der Hoffnung, dass dadurch ,überreicher Gewinn zu ziehen sein wird“ (Kausch 1903: 3), der wiederum eins zu eins mittels entsprechenden deoikonymischen Konstruktionsmustern abgebildet wurde.

Dass das koloniale Namenprojekt in der Metropole ausschließlich kommunikative Handlungsmacht der Kolonisatoren aufweist, kulminiert in den mit praxonymischen Anteilen verfügten toponymischen Konstruktionsmustern Tanga, Waterberg und Taku(-Fort) (Tab. 14). Dieser Befund kann mit den jeweiligen zeitgenössischen Zuschreibungen untermauert werden, wird aber bereits anhand der primären zeitgenössischen Intention der betreffenden MOD-Types deutlich: Die mit entsprechenden Konstruktionen vorgenommene Bezugnahme auf die aus deutscher Sicht erfolgreichen Kampfhandlungen konzentriert sich ausschließlich auf das Handeln und der damit verbundenen Herrschaftssicherung der deutschen Kolonialmacht. So war bspw. der Ort Tanga auf regional wirkende Funktionen als Handelszentrum beschränkt (vgl. Becher 1997: 168). Nichtsdestotrotz wurden dementsprechende Modifikator-Konstruktionen für kolonial motivierte Namenvergabepraktiken im Raum der deutschen Metropole als attraktiv angesehen, weil - das zeigen auch die bereits dargelegten Benennungsmotiviken zeitgenössischer Adressbücher (Kap. 7.1.2.2.c.) - sich die Namenverfügungen (auch) auf die „für unsere Schutztruppe so ruhm- und erfolgreiche[n] Schlacht von Tanga“ (Reichs-Kolonial-Amt 1915: 57) ${ }^{124}$ beziehen sollten, in der die deutschen Kolonialtruppen unter der Führung des Generals Lettow-Vorbeck die Briten besiegten.

Tanga war für die Briten ein Fiasko [und] [...] galt lange als eine der schlimmsten Niederlagen in den Annalen britischer Militärgeschichte. 800 Tote blieben auf britischer Seite auf dem Schlachtfeld, nahezu die Hälfte des britischen Offizierskorps war gefallen. Dagegen nahmen sich die Verluste der Deutschen gering aus. (Pesek 2010: 57)

Mit den Benennungspraktiken sollten Kampfhandlungen der deutschen Kolonialmacht in den kolonisierten Räumen (Tanga, Taku(-Fort)) oder die aus Sicht der deutschen Kolonisatoren erfolgreich niedergeschlagenen Aufstände der kolonisierten Bevölkerung (Waterberg), versprachlicht und im Raum der Metropole fixiert werden. Die mit praxonymischen Anteilen verfügten detoponymischen Konstruktionsmuster sollten damit die eigenen Verwaltungs- und Machtstrukturen in den kolonisierten Gebieten stabilisieren. Dieser Befund wird auch anhand der (bereits für deanthroponymische Benennungen aufgezeigten) per-

124 Teil der DSDK. 
sönlichen Bezüge innerhalb einzelner Benennungsmotiviken gestützt, die eine Verbindung rechtfertigen sollten:

Waterbergstraße: Am Waterberg, ehem. Deutsch-Südwestafrika, fand 1904 ein entscheidendes Gefecht im Hererofeldzug statt, an dem viele Bayern teilnahmen. (Adressbuch München 1941 IV: 313)

Groß-Nabas-Straße: Vom 2.-4.1.1905 fand bei Groß-Nabas in Deutsch-Südwestafrika ein Gefecht gegen den Großkapitän der Witboi-Hottentotten statt, an dem die Bayernkompanie hervorragend beteiligt war. (Adressbuch München 1941 IV: 313)

Im Gegensatz zu den kolonisierten Räumen, den dort errichteten Hauptorten und aus Sicht des Deutschen Kaiserreichs erfolgreichen Kampfhandlungen konnten dehydronymische und deoronymische Konstruktionen, die auf naturräumliche Gegebenheiten in den Kolonien referieren, nur für singuläre Städte des Deutschen Reichs erhoben werden. Die Fixierung solchen sprachlichen Wissens im (Alltags-)Raum der Metropole spielt eine zahlenmäßig völlig untergeordnete Rolle. Nichtsdestotrotz sind bei den Zuschreibungen in den enzyklopädischen Werken (Tab. 15) diskurslinguistisch interessante Beobachtungen festzustellen:

Tab. 19: Historische Zuschreibungen singulärer hydronymischer und oronymischer MOD-Types.

\begin{tabular}{|c|c|}
\hline MOD-Type & Zuschreibungen in Schnee (1920) und/oder Kausch (1903) \\
\hline Kilimandscharo & $\begin{array}{l}\text { von der Grösse des deutschen Harzes, höchster Berg Afrikas, landschaftliche } \\
\text { Schönheiten, einer der meisten genannten Landschaften der Kolonie, Kaiser- } \\
\text { Wilhelms-Spitze von Hans Meyer erstiegen, höchste Stelle Afrikas }\end{array}$ \\
\hline Usambara & der Küste am Nächsten liegender Teil \\
\hline Kibo & Höhenpunkt 5880 m, jetzt Kaiser-Wilhelmspitze \\
\hline Kongo & nächst dem Nil der längste Strom Afrikas \\
\hline Mohasi & einer der Seen des Ruanda \\
\hline Njassa & einer der großen Seen Ostafrikas \\
\hline Sambesi & einer der größten Ströme des Weltteils Afrika \\
\hline Tanganjika & auf ungefähr 670 km, Grenze zwischen Deutsch-Ostafrika und der Kongokolonie \\
\hline
\end{tabular}

In den Einträgen ist neben messbaren und/oder quantifizierbaren Angaben vor allem die Anzahl an Superlativen augenfällig, die man jenen naturräumlichen Gegebenheiten nicht nur in den zeitgenössischen wissensvermittelnden Texten des Deutschen Reichs, sondern auch den jeweiligen Namenverfügungen in 
entsprechenden Benennungsmotiviken der historischen Einwohnerbücher zugeschrieben hat:

Kibostraße: Kibo, 6010 m, höchster Gipfel des Bergmassivs des Kilimandscharo im Nordosten des früheren Ostafrika. (Adressbuch München 1941 IV: 350)

Das Wissen um das in faktischer Kolonialzeit in Deutsch-Ostafrika zu verortende Bergmassiv Kilimandscharo wurde sowohl durch entsprechende Alltagsliteratur (Reiseberichte, Zeitungstexte u. dgl.) als auch durch literarische Texte verbreitet: „Der Kilimandscharo verkörpert im spät zu Kolonien gelangten Deutschen Reich die neue Größe und besitzt dadurch als Kollektivsymbol einen besonderen Stellenwert im Alltagsbewusstsein“ (Hamann und Honold 2011: 122). In den enzyklopädischen Werken wird das Bergmassiv mit der „Grösse des deutschen Harzes“ (Kausch 1902: 54) gleichgesetzt - ein Vergleich, der auch in einer ganzen Reihe weiterer zeitgenössischer Texte und sogar in Geographieschulbüchern zu finden ist:

Wie eine Insel hebt sich das frischgrüne Kilimandscharo-Gebirgsland, das eine Fläche bedeckt, die den Harz bei Weitem übertrifft, aus der gelbbraunen Steppe heraus. (Lene 1908: 138)

Von hier aus erblickt man auch im fernen Osten, hoch über die Massaisteppe emporragend, den höchsten Berg von ganz Afrika, den zweigipfeligen Kilimandscharo, eine Bergmasse von 6010 m Höhe und einer Grundfläche, daß der ganze Harz bequem darauf stehen könnte. (Wünsche 1912: 3)

Im Jahrbuch über die deutschen Kolonien geht der Geograph Jäger (1914: 1) $)^{125}$ sogar einen Schritt weiter, wenn er behauptet, dass

jedes Schulkind [...] den Kilimandscharo so gut wie den Brocken oder die Schneekoppe oder den Montblanc [kennt]. Und mit dem Namen des Berges eng verknüpft ist der Name des kühnen Mannes, [...] dem wir es verdanken, daß dieser Berg ein deutscher Berg geworden ist, Hans Meyer.

Jene zeitgenössischen transtextuellen Aussagenzusammenhänge verdeutlichen, dass man auch mit dehydronymischen und deoronymischen Konstruktionen darauf abzielte, fremde Naturräume in den Kolonialgebieten als bekannte geographische Landschaften im öffentlichen Raum der Metropole als kollektive Wissensbestände zu fixieren. Dass mit derartigen Benennungen auch die uneingeschränkte Nutzbarmachung des kolonisierten Raumes durch die deut-

125 Teil der DSDK. 
schen Kolonisatoren versprachlicht wurde, verdeutlichen zeitgenössische Motiviken solcher Benennungen:

Usambarastraße: Usambara, Ort im ehem. deutschen Schutzgebiet Ostafrika an der Nordbahn gelegen, die Tanga und Moschi verbindet; berühmt durch die von deutschen Pflanzern geschaffenen Kaffeeplantagen. (Adressbuch München 1941 IV: 659)

Für den Teilbestand bleibt festzuhalten, dass nicht nur die Distributionen hinsichtlich der dabei verwendeten toponymischen Unterklassen erklärt werden können, sondern dass auch die damit versprachlichten kolonisatorischen Wissenskonzepte ein geradezu sprachlich-diskursives Muster offenlegen: Die in den Einträgen der enzyklopädischen Werke (vgl. Kausch 1903, Schnee 1920) für die entsprechenden Modifikator-Konstruktionen dargelegten Zuschreibungen, teilweise mit Einbezug der in den historischen Adressbüchern aufgezeigten Benennungsmotiviken der Inventare, verdeutlichen, dass mit derartigen Namenvergabepraktiken kein hochgradig komplexer, fremder Raum in den (Alltags-)Raum der deutschen Gesellschaft implantiert und sichtbar gemacht werden sollte. Die große Mehrheit der Benennungen bezieht sich auf den von Seiten des Deutschen Kaiserreichs erworbenen Kolonialbesitz, also auf die Kolonien und die dort von der Kolonialmacht als ökonomische und/oder administrative Zentren ausgebaute oder erst errichteten Hauptorte. Es wird also nicht auf den fremden Raum in Übersee referiert, sondern vorrangig auf die eigens errichteten kolonisierten Räume und die dort nach europäischem Vorbild errichteten Zentren. Selbst die zahlenmäßig völlig untergeordnete Zahl an deoronymischen und dehydronymischen Konstruktionen sollte auf keine wahllos ausgewählten Gebirge und Gewässer referieren, sondern bezieht sich auf aus Sicht der Kolonisatoren herausragende naturräumliche Gegebenheiten, die der uneingeschränkten Nutzbarmachung dienten oder denen teilweise sogar der symbolhafte Charakter eines Wahrzeichens zugesprochen wurde.

Auf dieses Ergebnis, dass mittels detoponymischer Konstruktionsmuster gerade die eigenen Geltungsansprüche versprachlicht wurden, soll im Folgenden ausführlicher eingegangen werden: Wenn zahlenmäßig vorrangig die eigenen Kolonien, die dort errichteten administrativen und/oder handelsspezifischen Zentren und die aus Sicht der Kolonisatoren erfolgreichen Kämpfe geehrt und/oder gewürdigt werden sollten, dann ist auch die Frage zu beantworten, auf welche kolonisierten Räume man sich mittels entsprechender Namenvergabepraktiken bezogen hat: Welche Kolonien spielten im kolonialen Projekt der Metropole eine vorrangige, welche dagegen offenbar nur eine marginale Rolle? Anhand der Annotation des Teilinventars an SN-Token mit toponymischen MODTypes nach den jeweils räumlichen Bezügen in Anlehnung an die historisch- 
räumliche Einteilung der Kolonialgebiete in den enzyklopädischen Werken (Kap. 5.2.4) werden nun die Distributionen räumlicher Georeferenz dargelegt, mit denen tradierte Gewissheiten über den kolonisierten Raum im (Alltags-) Raum der Metropole fixiert wurden.

\subsubsection{Räumliche Bezüge}

Die in Dresden und Breslau [Wrocław] verfügten Kolonialismen mit toponymischen MOD-Token zeigen nur ansatzweise eine Priorisierung räumlicher Bezüge auf: Wenngleich von acht MOD-Token drei SN-Token (Windhuker Straße und Swakopmunder Straße in Dresden, Windhukstraße in Breslau [Wrocław]) dem kolonisierten Raum in Deutsch-Südwestafrika zuzuschreiben sind (Kap. 5.7.2), ist daraus noch keine stichhaltige Hypothese für ortsübergreifend musterhafte Georeferenzierung im kolonialen Namenprojekt der deutschen Metropole abzuleiten. Dennoch ist zu konstatieren, dass die in beiden Städten verfügten detoponymischen Benennungen auf die Kolonien des Deutschen Kaiserreichs und auf Orte innerhalb der deutschen Kolonialgebiete referieren. Dieses erste Ergebnis bestätigt sich auch im inventarbezogenen Zugriff: Bis auf wenige Ausnahmen bezieht sich der Teilbestand an detoponymischen Konstruktionen ${ }^{126}$ auf den kolonisierten Raum des Deutschen Kaiserreichs:

Tab. 20: Distributionen toponymischer MOD-Token hinsichtlich räumlicher Bezüge.

\begin{tabular}{lll}
\hline Räumliche Unterscheidung & Deutscher Kolonialbesitz & $\begin{array}{l}\text { Kolonialbesitz anderer } \\
\text { europäischer Kolonialmächte }\end{array}$ \\
\hline Anzahl an MOD-Token & $\mathbf{1 7 8}$ & 7 \\
\hline
\end{tabular}

Das koloniale Namenprojekt im Raum der Metropole zeichnet sich also gerade dadurch aus, dass man vorrangig keine durch die europäischen Mächte erfolgte koloniale Raumunterwerfung darlegt, sondern sich fast ausschließlich auf den eigenen kolonisierten Raum beschränkt und durch entsprechende Benennungspraktiken im öffentlichen Raum fixiert, denn „der primäre Movens zu einem

126 In der Tabelle unberücksichtigt bleiben die SN-Token Kongo-, Mohasi-, Njassa-, Sambesi und Tanganjikastraße (mit singulären hydronymischen Modifikatoren), die auf naturräumliche Gegebenheiten (Gewässer) auf dem afrikanischen Kontinent referieren. Auch die SN-Token Pekinger Platz und das SN-Token Großfriedrichsburger Straße bleibt unberücksichtigt, weil die Modifikatoren weder dem faktischen Kolonialbesitz des Deutschen Kaiserreichs (1884-1919) noch dem der anderen europäischen Mächte unmittelbar zugeordnet werden können. 
Überseereich [bildete] die Gleichstellung mit anderen Kolonialmächten, insbesondere die Konkurrenz mit [...] Großbritannien“ (Berman 2003: 28). Die Benennungen sollten - das verdeutlichen ferner auch die fett markierten Possessiv- und Personalpronomen (unseres, uns) - den Kolonialbesitz des Deutschen Kaiserreichs und die innerhalb der deutschen Kolonien errichteten oder ausgebauten administrativen bzw. wirtschaftlichen Zenten ehren und/oder würdigen:

Kribiweg: Bezirkshauptort unseres Schutzgebietes Kamerun. (Adressbuch Bremen 1939 III: 162)

Samoaweg: Zur Erinnerung an die uns geraubte Kolonie Samoa. (Adressbuch Altenburg 1935 II: 190)

Tsingtauer Straße: Nach dem Hauptort des uns entrissenen ehemaligen deutschen Pachtgebietes in Ostasien. (Adressbuch Stuttgart 1940 III: 479)

Nur wenige Benennungsmotiviken der nach 1919 verfügten Straßennamen zeigen überhaupt die - aus zeitgenössischer deutscher Sicht ungerechtfertigte Abtretung der Kolonialgebiete an die Mandatsmächte auf. Nur für singuläre Benennungen in Einzelorten konnten entsprechende Hinweise erhoben werden:

Samoaweg: Ehemals Deutsches Schutzgebiet, kam nach dem Weltkrieg an England. (Adressbuch Frankfurt 1941 II: 305)

Togoweg: Ehem. Deutsche Kolonie in Westafrika, kam nach dem Weltkrieg an England und Frankreich. (Adressbuch Frankfurt 1941 II: 358)

Kamerunstraße: Kamerun, ehemaliges Deutsches Schutzgebiet in West-Afrika. Seit 1919 zwischen England und Frankreich (Mandatsgebiet) geteilt. (Adressbuch Köln 1937 IV: 364)

Togostraße: Ehemals kleinste deutsche Kolonie an der afrikan. Westküste [...] steht heute noch unter der Mandatsherrschaft Englands und Frankreichs [...]. (Adressbuch Köln 1939 IV: 749)

\section{a. Deutscher Kolonialbesitz: Distributionen}

Für den Hauptbestand aller detoponymischen Konstruktionen, die in räumlicher Hinsicht unmittelbar auf Orte in den deutschen Kolonialgebieten referieren (178 SN-Token, Tab. 20), wird der Frage nachgegangen, inwieweit räumliche Präferenzen hinsichtlich des zu ehrenden und/oder würdigenden kolonisierten Raumes festzustellen sind. Dabei wird auch der Frage nachgegangen, inwiefern die in zeitgenössischen Quellen als „Musterkolonien“"127 zu etablierenden Kolonien Togo, Kiautschou und Samoa auch quantitativ unter den jeweiligen

127 Man vgl. dazu 22 Treffer für Musterkolonie in der DSDK. 
choronymischen und oikonymischen Modifikator-Konstruktionen in der Metropole dominieren. So erklärte bspw. der in der Kolonie Togo tätige kaiserliche Regierungsarzt Külz (vgl. Schnee 1920 II: 390), dass man sich von Seiten der Kolonialverwaltung zum Ziel setzen müsse, die „Musterkolonie Togo vor den Augen der Heimat paradieren [zu] lassen [...]“ (Külz 1906: 93). ${ }^{128}$ Sie wird noch gegen Ende der faktischen Kolonialzeit als „Musterkolonie von heute“ (Trierenberg 1914: 202) ${ }^{129}$ beschrieben. Der Schriftsteller Reinecke, der am Ende des 19. Jahrhunderts eine fünfjährige Forschungsreise nach Samoa unternommen hatte, forderte für die im Pazifischen Ozean liegende Kolonie DeutschSamoa eine ähnliche Präsenz im Kaiserreich:

[...] wenn uns die „Perle der Südsee“ den langersehnten Beweis liefern soll, dass sie des Schweisses [sic!] der Edlen wert ist und sich als Musterkolonie bewährt. Dazu aber muss auch das Mutterland und unsere Kolonialverwaltung zunächst den lange vorenthaltenen richtigen Beistand gewähren und zunächst für eine Festigung der Bande mit der Heimat, für gute Schiffsversbindungen sorgen. (Reinecke 1902: 254) ) $^{130}$

Für die von Seiten der deutschen Kolonialverwaltung umgesetzte Schul- und insbesondere Kulturpolitik stellt die „Deutsche Vereinigung Schanghai“ (1913: 93) ${ }^{131}$ in ihrer „Denkschrift zur Förderung des Deutschtums in China“ als werbende Selbstzuschreibung heraus, dass die Stadt „Tsingtau [...] eine Musterkolonie geworden ist“. Daneben konnten aber auch weitere historische Quellen gesichtet werden, in denen Tsingtau als mustergültig beschrieben wird: So zeigt bspw. Vollerthum (1924: 66) auf, dass der „Ruf Tsingtaus als Musterstätte deutscher Kultur“ auch nach der Abtretung der Handelskolonie weiterhin verbreitet wurde. Das Diagramm zeigt die Distributionen auf, die sich durch entsprechende Annotation detoponymischer Konstruktionen ergeben:

$\mathbf{1 2 8}$ Teil der DSDK.
$\mathbf{1 2 9}$ Teil der DSDK.
$\mathbf{1 3 0}$ Teil der DSDK.
$\mathbf{1 3 1}$ Teil der DSDK. 


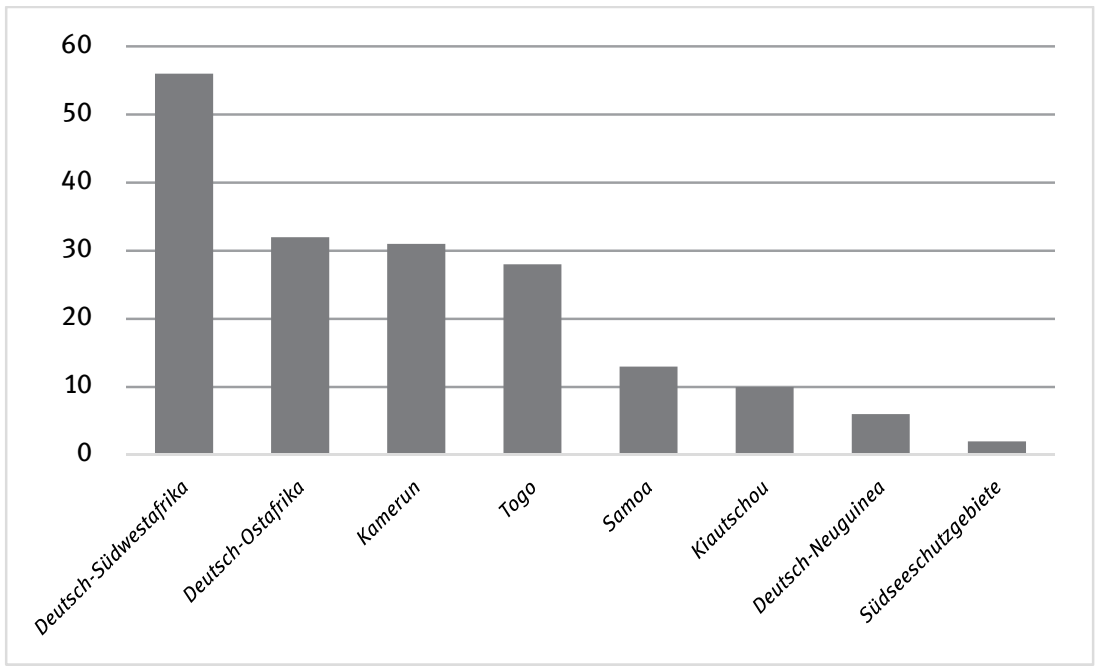

Diagramm 3: Georeferenzierende Bezüge toponymischer MOD-Token.

Die sich dabei ergebende Häufigkeitsverteilung zeigt, dass bei dem kolonialen Namenprojekt der Metropole keine zahlenmäßig dominante Kommemoration der als sogenannte Musterkolonien zu etablierenden Kolonien Togo, Kiautschou und Samoa festgestellt werden kann. Stattdessen ist eine übergeordnete räumliche Referenz auf die afrikanischen Kolonien durch nahezu 150 Straßenbenennungen festzustellen. Dabei erweist sich die Integration der Kolonie DeutschSüdwestafrika in den (Alltags-)Raum des Deutschen Reichs als zahlenmäßig dominant: Für über 50 SN-Token sind georeferenzierende Bezüge auf DeutschSüdwestafrika auszumachen. Welche Konstruktionen dabei maßgebliche Verwendung fanden, wird im Folgenden näher erläutert.

\section{b. Spezifika: Deutsch-Südwestafrika}

Folgende toponymische MOD-Types wurden als zweigliedrige Konstruktionen ortsübergreifend im Raum der deutschen Metropole verfügt:

Tab. 21: Toponymische MOD-Types (ortsübergreifend), Deutsch-Südwestafrika.

\begin{tabular}{llllll}
\hline MOD-Types & Windhuk & Swakopmund & Waterberg & Otavi & (Deutsch-)Südwest(-Afrika) \\
\hline Anzahl an SN-Token & 25 & 10 & 10 & 6 & 4 \\
\hline
\end{tabular}


Innerhalb jenes Teilbestandes an ortsübergreifend verfügten Namenvergabepraktiken, die eine Kommemoration des kolonisierten Raumes Deutsch-Südwestafrikas vornehmen sollten, ist der Hauptanteil deoikonymischer Konstruktionsmuster herauszustellen, die sich auf den Hauptort der Kolonie (Windhuk), die wichtigste Küstenstadt (Swakopmund) und den Handelsknotenpunkt im Inland (Otavi) beziehen. Das mit der Otavibahn transportierte Kupfererz konnte nach Swakopmund zur Verschiffung nach Europa befördert werden (vgl. Hofmann 2013: 103). Während des Ersten Weltkriegs gewann Otavi und der kolonisierte Raum in Deutsch-Südwestafrika aufgrund des Kupfers, das eines der wichtigsten Bergwerksprodukte für die deutsche Kriegsindustrie darstellte, eine wichtige Rolle, wie folgende Ausführungen des Kolonial-Wirtschaftlichen Komitees (ca. 1915: 2) ${ }^{132}$ zeigen:

\begin{abstract}
Während des Krieges ist die deutsche Kupferproduktion in erheblichem Maße gesteigert worden; aber selbst wenn sie in der Folge wird auf gleicher Höhe gehalten werden können, ist kaum anzunehmen, daß die Steigerung mit dem Wachstum unseres Verbrauchs gleichen Schritt halten wird. [...] Hilfe kann uns nur vom Kolonialbesitz kommen, und von unseren Kolonien war es besonders Südwestafrika [Fettdruck im Original], das bereits nennenswerte Mengen Kupfererze ausführte. [...] Die Hauptmenge lieferten die bekannten Otaviminen.
\end{abstract}

Neben Benennungen mit dem MOD-Type Waterberg, für den neben toponymischen Referenzen primär praxonymische Bezüge nachgewiesen wurden (Kap. 7.1.2.2 c.), konnten nur vier Straßenbenennungen erhoben werden, die in kommemorativer Intention auf das entsprechende Choronym ${ }^{133}$ referieren. Das könnte auch an formalen Vorgaben liegen, die bei administrativen Straßenvergabepraktiken zu berücksichtigen sind. Laut des „Runderlaß[es] des Ministers des Innern“ (17.06.1933: 745-746) sollte „der ganze Name einschließlich des Beiworts [...] in der Regel nicht mehr als fünf Silben haben und höchstens aus zwei getrennten Wörtern bestehen“, was auch die Verfügung von SN-Token wie Deutsch-Südwestafrika-Straße verboten hätte. Es kann nur vermutet werden, dass die seltenen Fälle solcher dechoronymischer Konstruktionen damit zusammenhängen, dass gerade die zulässigen abgekürzten Formen (Deutsch-) Südwest von den städtischen Administrationen als zu unspezifisch angesehen wurden und aus diesem Grund nicht attraktiv genug oder nicht möglich erschienen. Auch weiteren Benennungen sind georeferenzierende Bezüge auf

132 Teil der DSDK.

133 D. s.: Deutsch-Südwestafrika-Straße in Stuttgart; Südweststraße in Bremen; Südwestkamp in Hamburg; Südwester Straße in Lodz bzw. Litzmannstadt [€ódź]. 
Deutsch-Südwestafrika zuzusprechen, die allerdings nur einzelortsspezifische Verwendung fanden (u. a. Groß-Nabas-Straße, Lüderitzlandstraße).

Die Präferenz einer räumlichen Bezugnahme auf die Kolonie DeutschSüdwestafrika, die durch entsprechende Namenvergabepraktiken in den Raum der deutschen Metropole fixiert wurde, ist hinsichtlich der damit versprachlichten Gewissheiten zu diskutieren: Frühe positive Berichterstattung über DeutschSüdwestafrika erfolgte in politischen Zeitschriften des Kaiserreichs, die sich unter anderem auch mit der Kolonialherrschaft in Übersee beschäftigten. In der Zeitschrift Die Grenzboten (1892: 175) freute man sich bspw. insbesondere über die Erfolge der christlichen Missionen im ,älteste[n] unsrer jungen Schutzgebiete [...]. In keinem andern afrikanischen Lande hat die Mission [...] so tüchtig vorgearbeitet wie hier. Fast die ganze Bevölkerung ist bereits christlich“. Neben der in der Kolonie verfügbaren „Bergschätze [...] und ihre[r] Ausbeute [...] für die heimische Industrie“ (Kolonial-Wirtschaftliches Komitee ca. 1915: 3) ist außerdem noch ein weiterer möglicher Beweggrund zu erläutern, der die Bevölkerungszusammensetzung in Deutsch-Südwestafrika in der faktischen Kolonialzeit betrifft: Fünf Jahre nach dem „schwerste[n] Eingeborenenaufstand im Schutzgebiet Deutsch-Südwestafrika seit der Besitzergreifung“ (Schnee 1920 II: 59) wird die Kolonie von Seiten des Staatssekretärs des Reichs-Kolonialamts als „unserer blutgetränkten und vielgeschmähten, aber auch dem Herzen des ganzen Volks liebgewordenen Siedlerkolonie“ (Dernburg 1909: 11) ${ }^{134}$ beschrieben. Auch in weiteren zeitgenössischen Quellen wird Deutsch-Südwestafrika als einzige Siedlungskolonie der deutschen Kolonialmacht verhandelt, in der „mit einem Anteil von $56 \%$ deutlich mehr als die Hälfte aller deutschen Reichsangehörigen [...] in den Kolonien zum Ende der deutschen Herrschaft in DeutschSüdwestafrika ansässig war“ (Stolz und Warnke 2018c: 87). Die Anzahl der deutschen Bevölkerung in Deutsch-Südwestafrika lag vor Beginn des Ersten Weltkriegs im fünfstelligen Bereich, was bereits in der zeitgenössischen Enzyklopädie von Schnee (1920) durch entsprechende Einträge dargelegt wurde:

Bevölkerung der Schutzgebiete [Fettdruck im Original] In den Schutzgebieten hat die weiße Bevölkerung entsprechend dem wirtschaftlichen Aufschwung, den die Kolonien genommen haben, eine bedeutende Zunahme erfahren. [...] und verteilt sich folgendermaßen: Deutsch-Ostafrika 5336 [...] (darunter Deutsche 4107); Kamerun 1871 [...] (darunter Deutsche 1643); Togo 368 [...] (Deutsche 320); Deutsch-Südwestafrika 14830 (Windhuk 2871, Lüderitzbucht 1616, Swakopmund 1463) (Deutsche 12292); Deutsch-Neuguinea I (Kaiser-Wilhelmsland und Bismarckarchipel) 968; Deutsch-Neuguinea II (Karolinen, Marshallinseln) 459 (Deutsche 1005); Samoa 557 (Deutsche 329). (Schnee 1920 I: 195-196)

134 Teil der DSDK. 
Siedlung in den Schutzgebieten [Fettdruck im Original] Die Frage der kolonialen Siedlung im engeren Sinne, d.h. der dauernden generationsweisen Niederlassung von Weißen, besonders Volksgenossen, in den Kolonien zu wirtschaftlicher, speziell landwirtschaftlicher, Tätigkeit ist bei uns in Deutschland mehr und mehr eine parteipolitische Frage geworden [...]. Unter den deutschen Schutzgebieten in Afrika und der Südsee kommt nun als Siedlungsgebiet gegenwärtig unbestritten nur Deutsch-Südwestafrika in Betracht. [...] Großes Gewicht auf eine rein sachliche Beurteilung der Siedlungsmöglichkeit von Fall zu Fall unter Berücksichtigung der wirtschaftlichen und soizalen [sic!] neben den natürlichen Verhältnissen legte der derzeitige Staatssekretär des Reichs-Kolonialamts Dr. Solf in seiner Reichstagsrede vom 9. März 1914. Auch er rechnete zu den unbestrittenen Siedlungskolonien nur Südwestafrika [...]. (Schnee 1920 III: 352)

Strukturelle Besonderheiten makro- und mikrotoponymischer Benennungspraktiken des kolonialen Namenprojekts in Deutsch-Südwestafrika selbst wurden bereits in kolonialtoponomastischen Schriften aufgezeigt: Für den in DeutschSüdwestafrika erhobenen makrotoponymischen Bestand haben Stolz und Warnke (2018c: 88) herausgestellt, dass „,verstärkt direkte Übernahmen aus dem reichsdeutschen Toponymikon auf[treten] ${ }^{\star 135}$; sie sind damit zu erklären, dass

[...] es für die Ortsnamenvergabe die persönliche Verbundenheit eines Individuums mit der namengebenden reichsdeutschen Gemeinde gab. Es wird der Name des Geburtsortes eines sich als Farmer niederlassenden deutschen Siedlers gewählt. [...] Die persönliche Note, die im deutsch-südwestafrikanischen Toponymikon eine große Rolle spielt, wird noch deutlicher, wenn wir die Verwendung von Personennamen in toponymischer Funktion in Betracht ziehen. (Stolz und Warnke 2018c: 89-90)

Auch die in zeitgenössischen Quellen für Deutsch-Südwestafrika erhobenen Namen unterhalb der Ortsebene zeichnen sich häufig durch die Übernahme reichsdeutscher Mikrotoponyme aus (vgl. Aleff 2017: 20). Jene "Südwester Spezifika“ (Stolz und Warnke 2018c: 88) sind aus sprachhistorischer Perspektive offenbar damit zu erklären, dass sowohl deutsche Privatpersonen als auch die deutsche Kolonialadministration in gewissem Rahmen einen für die „weiße Bevölkerung“ (Schnee 1920 I: 195) vertrauten öffentlichen Raum konzipieren wollte.

Während für Deutsch-Südwestafrika insbesondere aufgrund ihres zeitgenössischen Charakters als einzig möglicher Siedlungskolonie die Übernahme reichs-deutscher Makrotoponyme und reichs-deutscher Mikrotoponyme für die jeweiligen Hauptorte festzustellen sind, wurden kolonialdeiktische Wissens-

135 Bspw. Brandenburg, Bremen, Frankfurt oder Karlshorst. Ortsnamen außerhalb DeutschSüdwestafrikas stellen dagegen mehrgliedrige Konstruktionen dar, bspw. Neu-Trier und Mecklenburg-Bucht in Deutsch-Ostafrika bzw. Neu-Pommern und Berlin-Reede in Deutsch-Neuguinea (vgl. Stolz und Warnke 2018c: 88-89). 
konzepte über die Kolonie Deutsch-Südwestafrika wiederum durch hochfrequente Straßennamenvergabepraktiken im (Alltags-)Raum des Deutschen Reichs fixiert. Auf die in der unmittelbaren Raumdeskription sichtbaren Auswirkungen der deutschen Kolonialzeit und der damit einhergehenden partiellen Beibehaltung reichs-deutscher Benennungen von Straßen, Plätzen, Gebäuden u. dgl. wird sogar in Benennungsmotiviken kolonialer Namenverfügungen im Raum der deutschen Metropole hingewiesen. Für das in nationalsozialistischer Zeit verfügte SN-Token Swakopmunder Straße in München ist folgender Eintrag im zeitgenössisch für die städtische Öffentlichkeit verbreiteten und stark rezipierten Einwohnerbuch festzustellen:

Swakopmunder Straße: Swakopmund, heute noch vollkommen deutsche Stadt an der südwestafrikanischen Küste. (Adressbuch München 1941 IV: 619)

Mit dem expliziten Hinweis auf die vollkommen deutsche Stadt werden implizit die noch Anfang der 1940er Jahre zu konstatierenden Auswirkungen der deutschen Kolonialzeit auf das seit 1920 als Mandatsgebiet des Völkerbundes zugehörende South West Africa verdeutlicht.

\section{c. Kolonialbesitz anderer europäischer Kolonialmächte}

Weniger als zehn toponymische MOD-Types zeigen räumliche Bezüge auf, die dem Kolonialbesitz anderer europäischer Mächte zuzuschreiben sind. Für koloniale Fixierungspraktiken in der deutschen Metropole stellten derartige Benennungen ein zahlenmäßig untergeordnetes Phänomen dar, das sich auf wenige Städte beschränkt. Nur Konstruktionen mit dem MOD-Type Sansibar, dessen Benennungen auf das gleichnamige Insel-Sultanat als Teil des britischen Kolonialbesitzes (Tab. 12) referierten, konnten für den Untersuchungszeitraum drei Mal erhoben werden. Alle weiteren MOD-Types sind nur für Berlin-Wedding ${ }^{136}$ (Senegalstraße, Ugandastraße) oder durch maximal zwei Konstruktionen (Transvaalstraße in Berlin-Wedding und Oranienburg) im erstellten Inventar vertreten. Überhaupt beschränken sich derartige Namenvergabepraktiken auf die Städte Berlin, München und Oranienburg. Im Hinblick auf die damit intendierten diskursfunktionalen Dimensionen wurde für den MoD-Type Transvaal die zeitgenössische Funktion jener Provinz bereits in Kap. 4 dargelegt: Wenngleich Transvaal nie in deutschem Kolonialbesitz war, konnte unter Einbezug zeitgenössischer Quellen verdeutlicht werden, dass die Provinz, unter anderem durch die hohe Zahl an deutschen Siedlern, ein Ort kolonialer Imaginationen darstellte. Auch der MOD-Type Sansibar scheint hinsichtlich der Funktionen, die

136 Senegal-, Kongo- und Ugandastraße. 
man mit den ortsübergreifenden Namenvergabepraktiken intendierte, nur auf den ersten Blick überraschend: Stolz und Warnke (2018b: 56-60) haben für den makrotoponymischen Bestand deutscher Kolonialtoponyme im kolonisierten Raum bereits festgestellt, dass es sich bei der im Indischen Ozean liegenden Insel um ein „diskurserschließendes Toponym“ handelt, das im Kontext deutschkolonialer Diskursdaten ${ }^{137}$ breite Verwendung fand:

Zanzibar/Sansibar ist sicher nicht nur wegen seines endonymischen Exotismus ein diskurserschließendes Toponym, sondern auch wegen seiner kontrafaktischen Semantik. Es hält sich bis heute hartnäckig die Legende, dass das Deutsche Reich 1890 in einem Vertrag mit dem Vereinigten Königreich Sansibar gegen Helgoland getauscht habe. Der Anspruchsverzicht ist aber einmal mehr ein diskursförderndes Ereignis. (Stolz und Warnke 2018b: 60)

Dass „Sansibar [kursiv im Original] als Top gerade durch den Verzicht auf geopolitische Ansprüche ein Emblem für koloniale Imaginationen zu sein“ (Stolz und Warnke 2018b: 60) scheint, wird auch durch die in den enzyklopädischen Werken vorgenommenen Zuschreibungen deutlich. So wird bspw. in Schnee (1920 I: 285, Tab. 13) für das Lemma Daressalam angegeben, dass der Hauptort DeutschOstafrikas „unter allen guten Hafenorten der ostafrikanischen Küste derjenige [ist], der Sansibar, dem ehemaligen Schlüssel des tropischen Ostafrika, am nächsten liegt.“ Dass jene Legende sogar zu den noch in nationalsozialistischer Zeit tradierten Gewissheiten gehört, zeigt die im zeitgenössischen Einwohnerbuch aufgeführte Motivik der Mitte der 1930er Jahre in München-Trudering verfügten Sansibarstraße:

Sansibarstraße: Sansibar, Inselgruppe gegenüber Deutsch-Ostafrika durch Vertrag vom 1.7.1893 im Austausch gegen Helgoland an England abgesetzt. (Adressbuch München 1941 IV: 552)

Wenngleich es sich bei Namenverfügungen, die auf den Kolonialbesitz anderer europäischer Kolonialmachte referieren, um ein eher randständiges Phänomen handelt, sind kritische Stellungnahmen von Seiten kolonialer Interessengruppen nicht ausgeblieben: So kommentierte die DKG die 1912 von Seiten der Administration gewählten Benennungen Kongo- und Sansibarstraße für zwei neue Straßenzüge im Zuge der fortwährenden Erschließung des Weddinger Wohnbezirks folgendermaßen:

137 Hierfür wurde das Toponym Zanzibar/Sansibar innerhalb des Referenzkorpus DSDK systematisch kontextualisiert. 
Koloniale Strassenbezeichnungen [Fettdruck im Original]. In der Reichshauptstadt sind vor kurzem zwei neu entstandene Straßen Kongostraße und Sansibarstraße genannt worden. Wir wollen mit der Berliner Verwaltung nicht rechten, daß sie einen afrikanischen Strom, an den wir gerade heranreichen, und eine Insel, die als fremder Besitz unserem größten Schutzgebiete vorgelagert ist, ${ }^{138}$ zur Namengebung benutzt hat, weil die uns näherliegenden kolonialen Bezeichnungen bereits im Berliner Straßengewirr in Erscheinung treten. Es gibt eine Togostraße, eine Kameruner-, Kiautschou-, Guinea- und Lüderitzstraße. (DKZ 18.1.1913: 35)

Die DKG legt dar, dass die kolonialen Benennungen bereits in der faktischen Kolonialzeit einer zeitlichen Schichtung unterlagen. Dies ist Ausgangspunkt dafür, dass die nicht unkritische Stellungnahme hinsichtlich jener Benennungen, die sich auf den Kolonialbesitz anderer europäischer Kolonialmächte beziehen, zu einem gewissen Anteil revidiert wird: Die aus Sicht der DKG administrativen Namenverfügungen im schon zur Kolonialzeit als „Kolonialviertel“ (Winzer 1907/10: 2) bezeichneten Weddinger Baulands sind insofern annehmbar, als dass bereits Benennungen mit „näherliegenden kolonialen Bezeichnungen“ mit anthroponymischen (Lüderitzstraße) und choronymischen (Kameruner Straße; Kiautschou-, Guinea- und Togostraße) MOD-Types erfolgten. Der Text zeigt also gleichzeitig auch zeitgenössische Gewissheiten kolonialer Interessengruppen in Bezug auf koloniale Namenvergabepraktiken auf. Dass die Kolonialbewegung zwischen 1884 und 1945 nicht nur koloniale Straßenvergabepraktiken forderte, sondern ihr auch ein maßgeblicher Einfluss auf die Konstruktionsmuster zugesprochen werden muss, die im kolonialen Namenprojekt in der Metropole Verwendung fanden, soll im Folgenden näher erläutert werden.

138 D. i. Kamerun; man vergleiche hierzu den Lexikoneintrag zu Kamerun in Schnee (1920 II: 169): „Nur mit dem Kongozipfel würden wir den belgischen Kongostaat berühren, wenn nicht die Inseln im Kongo französisch geblieben wären. Unser Gebiet umfaßt nach neuen Feststellungen des geodätischen Bureaus im KA. rund 795000 qkm; davon haben wir durch das Abkommen vom 4. Nov. 1911 mit Frankreich (s. Erwerb der deutschen Kolonien 3) 295000 qkm gewonnen, aber mit dem sog. Entenschnabel 12000 verloren. Was nun die Grenzen von K. anbetrifft, so sind dieselben teilweise noch nicht festgelegt“. 


\title{
7.4 Strukturmuster und Diskursfunktionen: Einfluss durch Gruppen der Kolonialbewegung
}

Dass die DKG als einflussreichster Verband der Koloniallobby (vgl. Heyden und Zeller 2002: 12) schon in der faktischen Kolonialzeit eine genaue Vorstellung davon hatte, welche Benennungen man als „geeignet [hielt], der kolonialen Sache zu dienen“ (DKZ 18.1.1913: 35), wird anhand folgender appellierender Mahnung deutlich:

\begin{abstract}
Nun ist bekannt, wie schwierig es für die Verwaltungen unserer stetig sich ausdehnenden Großstädte ist, die neu entstehenden Straßenzüge zu benennen. Warum werden nicht koloniale Bezeichnungen verwendet? Für die koloniale Sache wäre es doch ein Gewinn, wenn unsere Jugend zwischen einer Togo-, Duala-, Windhuk-, Lüderitz-, Wissmannstraße sich bewegte und so von früh auf lernet, daß diese Namen [...] Plätze des überseeischen Deutschlands und der Helden [sind], die an seinem Aufbau mitgewirkt haben. (DKZ 18.1.1913: 35)
\end{abstract}

Die von Seiten der DKG eingeforderte Kommemoration von „Plätze[n] [...] des überseeischen Deutschlands und der Helden, die an seinem Aufbau mitgewirkt haben" mittels entsprechender Straßenvergabepraktiken wird durch die Nennung konkreter Konstruktionsmuster unterstützt. Die von Seiten der Kolonialbefürworter dargelegten choronymischen, oikonymischen und anthroponymischen Modifikator-Konstruktionen Togo, Duala, Windhuk, Lüderitz und Wissmann gehören $\mathrm{zu}$ den ortsübergreifend verfügten 27 MOD-Types, die in der Verbindung usueller Klassifikatoren bis 1945 in den öffentlichen städtischen Raum des Deutschen Reichs verfügt wurden (Diagramm 1). Der Einfluss der Kolonialbewegung auf kolonial motivierte Namenvergabepraktiken und die hierfür auszuwählenden Konstruktionen ist demnach nicht erst im Zuge der Gleichschaltung der kolonialen Verbände, Vereinigungen und Gesellschaften unter nationalsozialistischer Führung in den 1930er Jahren als Reichskolonialbund (vgl. Schulte-Varendorff 2006: 110-111), sondern bereits seit der faktischen Kolonialepoche in der Zeit des Deutschen Kaiserreichs festzustellen. Dass die DKG kolonial motivierte Straßennamenverfügungen auch nach der de factoHerrschaft in der Zwischenkriegszeit einforderte, zeigt ihre am Anfang der 1920er Jahre erfolgte Bitte an den Städtetag:

Benennung von Straßen und Plätzen. Die Deutsche Kolonial-Gesellschaft hat sich auf Grund eines Beschlusses der Hauptversammlung an den Städtetag gewandt mit der Bitte, den Städten zu empfehlen, geographische Namen aus den deutschen Schutzgebieten zur Benennung von Straßen und Plätzen zu verwerten. (Deutscher Städtetag 21.10.1922) 
Nur für eine überschaubare Anzahl an Orten (Tab. 7) sind die jeweiligen städtischen Administrationen dem Wunsch der DKG in der Zwischenkriegszeit nachgekommen. Ein maßgeblicher Einfluss der Kolonialbewegung ist aber insofern zu konstatieren, als dass erstmalig ortsübergreifend verfügte Konstruktionen mit choronymischen und oikonymischen MoD-Token (Kamerun, Togo, Windhuk) festzustellen sind (Tab. 9). Obwohl koloniale Straßenbenennungen nur für einzelne (Groß-)Städte erhoben werden konnten, beziehen sich die ortsübergreifend innerhalb dieses Zeitraums verfügten Namen nicht nur auf Kolonialakteure. Stattdessen sind maßgeblich auch „geographische Namen aus den Schutzgebieten“ (Deutscher Städtetag 21.10.1922) vertreten, deren jeweilige Konstruktionen die Anzahl an verfügten deanthroponymischen Konstruktionen sogar überragt.

In nationalsozialistischer Zeit sind Forderungen nach kolonial motivierten Namenvergabepraktiken von staatlicher Seite konkretisiert worden. In der „Ausführungs-Anweisung zur Verordnung über die Benennung von Straßen, Plätzen und Brücken“ (Runderlaß des Reichsministers des Innern 15.07.1939) wird die Benennungskompetenz den Bürgermeistern (in Abstimmung mit den Ortspolizeibehörden) zugesprochen. Zur Benennung von Straßen sollen neben „[...] Namen der Länder, Städte und Ortschaften [...] des Deutschen Reichs und der deutschsprachigen Gebiete des Auslandes“ auch die

deutschen Kolonien, ferner die Namen von Orten, an die sich besondere Ereignisse (Schlachten oder dergleichen) knüpfen, in Betracht [kommen]. Des weiteren sind die Namen von Männern der deutschen Geschichte zu wählen, insbesondere von Vorkämpfern der nationalsozialistischen Weltanschauung, von großen Staatsmännern, Heer- und Flottenführern, von Männern, die sich im Kriege, bei der nationalsozialistischen Erhebung sowie im Kampfe um das deutsche Volkstum besonders ausgezeichnet haben [...]. (Runderlaß des Reichsministers des Innern 15.07.1939)

Bereits zuvor sind zeitgenössische Anfragen lokaler Abteilungen der DKG bzw. des in der Mitte der 1930er Jahre gegründeten Reichskolonialbundes (RKB) zu verzeichnen, die mit entsprechenden Anliegen die städtischen Administrationen zu kolonial motivierten Namenvergabepraktiken aufforderten. So zeigen Grewe et al. (2018: 131) für Freiburg auf, dass die Oberbadische Abteilung der DKG sich mit einer entsprechenden Bitte an den Freiburger Oberbürgermeister wandte:

Wiederholt gab es Mitte der 1930er Jahre Anfragen seitens der Kolonialvereine [...]. 1936 erklärte OB [d. i. Oberbürgermeister] Kerber, die Stadt sei grundsätzlich bereit, Straßen und Plätze nach alten Kolonialgebieten oder deutschen Kolonialpionieren zu benennen (Tangaplatz, Karl Petersstrasse, Lüderitzstraße u.s.w. [kursiv im Original])

Obwohl es aufgrund planerischer Gründe in Freiburg nicht zur Umsetzung der vorgeschlagenen Namen kam, liegt eine historische Beschreibung vor, wie eine 
Einzelperson - vermutlich auf die Namenvorschläge der DKG Bezug nehmend Mitte der 1930er Jahre das Thema konturiert. Auch die Namenvorschläge des RKB decken sich mit derartigen Konstruktionen. So erfolgten Empfehlungen des Chemnitzer Kreisverbandes (27.8.1936: 2), der die Interessen des RKB auf lokaler Ebene vertrat, an den Chemnitzer Oberbürgermeister:

Straßenbenennung nach deutschen Kolonialpionieren. [Unterstreichung im Original] Was diesen Punkt anbelangt, sieht es in unserer Stadt leider schlecht aus. [...] Um weiteste Kreise auch der Chemnitzer Bevölkerung auf die koloniale Frage aufmerksam zu machen und auch in ihnen das Verlangen nach Kolonien wachzurütteln, schlage ich - zugleich im Auftrage des Führers des Reichskolonialbundes [...] dem Herrn Oberbürgermeister vor, [...] Straßen und Plätze nach verdienten deutschen Kolonialpionieren umzubenennen.

Hierfür schlug der Kreisverband die SN-Token Lüderitzstraße, Wißmannstraße, Karl-Peters-Straße, Lettow-Vorbeck-Straße, Gustav-Nachtigal-Straße und Tangaplatz vor (vgl. RKB, Kreisverband Chemnitz 27.8.1936: 3). Für die Verfügung des SN-Tokens Karl-Peters-Straße hat sich der Chemnitzer Kreisverband (27.08.1936: 3) in besonderem Maße eingesetzt:

Dr. Karl Peters hat es als erster verdient, daß sein Name in jeder deutschen Stadt verewigt wird. Ihm haben wir es zu verdanken, daß das Vorkriegsdeutschland eine Kolonialmacht wurde, und gerade ihn hat das deutsche Volk - besser der deutsche Reichstag - so schmählich und bar jeden gesunden Instinktes behandelt. Da sich sein Geburtstag am 27. September zum 80. Male jährt, ist der Zeitpunkt für die nachträgliche Würdigung seiner Verdienste günstig gelegen. Ich darf der Erwartung Ausdruck geben, daß sich die Stadt Chemnitz dem Vorbild vieler Städte anschließen wird und den deutschen Kolonialpionieren der Vergangenheit in der von mir vorgeschlagenen Weise den Dank abstattet.

Auch die für Chemnitz empfohlenen Straßennamen wurden nicht umgesetzt. ${ }^{139}$ Nichtsdestotrotz zeigen die Vorschläge jener beiden ortsbezogenen Beispiele Namenmuster mit identischen Modifikator-Konstruktionen auf: Sowohl bei den für Freiburg als auch für Chemnitz festzustellenden Namenempfehlungen werden die MOD-Types Lüderitz, Peters und Tanga (neben weiteren) als offenbar prototypisch verstandene kolonial motivierte Konstruktionen im Nationalsozialismus verhandelt, die in hochfrequenter Anzahl mit entsprechenden usuellen Klassifikatoren in den öffentlichen städtischen Raum des nationalsozialistischen Deutschen

139 Derartige Empfehlungen sind auch für weitere Städte des Ortskorpus festzustellen: So kann bspw. für Augsburg nachgewiesen werden, dass der hiesige Gauverbandsleiter, angeregt durch den Reichskolonialbund, vorschlug, verschiedene Straßen in Augsburg mit kolonialer Motivik zu benennen (Apia-, Duala-, Kamerun-, Lome-, Lüderitz-, Peters-, Samoastraße u. dgl.). Herzlicher Dank geht an G. Feuerer vom Stadtarchiv Augsburg. 
Reichs verfügt wurden: Die anthroponymischen MOD-Types Peters und Lüderitz stellen hochfrequente Muster kolonial motivierter Straßenbenennungen zwischen 1933 und 1945 dar. ${ }^{140}$ Aus zeitgenössischen Akten geht hervor, dass der RKB sich sogar auf nationaler Ebene am Ende der 1930er Jahre für die „Benennung von Straßen und Plätzen mit den Namen bewährter Kolonialkämpfer“ mittels eines Rundschreibens der RKB-Bundesleitung eingesetzt hat, was sodann an die jeweiligen regionalen Gauverbände weitergeleitet wurde. So ist bspw. die Straßenbenennung Karl-Peters-Straße in Iserlohn auf Unternehmungen des RKBs zurückzuführen, die vom RKB-Ortsverbandsleiter Gauverband Westfalen Süd an den Iserlohner Bürgermeister herangetragen wurde:

Sehr geehrter Herr Bürgermeister! Die Bundesleitung des Reichskolonialbundes hat mich durch Rundschreiben beauftragt, mich bei den zuständigen Stellen dafür zu verwenden, dass bei der Benennung von Strassen und Plätzen Namen bewährter Kolonialkämpfer Verwendung finden. Ich wende mich [...] an Sie mit der Bitte, dieser Anregung bei erster Gelegenheit Rechnung zu tragen. Mit Vorschlägen stehe ich Ihnen jeder Zeit gern zu Diensten. Ich denke dabei in erster Linie an den in Iserlohn nicht unbekannten Dr. Karl Peters, welcher sich für die kolonialen Belange Deutschlands grosse Verdienste erworben hat. (Schreiben des Reichskolonialbundes, Gauverband Westfalen Süd 19.5.1938)

Darüber hinaus sind auch ortsübergreifende Benennungen mit dem MOD-Type Tanga erst für die NS-Zeit auszumachen, in der entsprechende Konstruktionen in 14 Groß- und Mittelstädten Verwendung fanden. Der „Schlacht bei Tanga“ (Mader 1938) ${ }^{141}$ wurde nicht nur publizistische Bedeutung verliehen, sondern auch durch Namenvergabepraktiken in den öffentlichen deutschsprachigen Raum. Der Kolonialkrieg wurde schon in der unmittelbaren Nachkriegszeit von der Kolonialbewegung für propagandistische Zwecke genutzt:

Am nachhaltigsten war der deutsche Widerstand in Ostafrika [...], wo die Verteidigung in der Person des Generals von Lettow-Vorbeck einen besonders vortrefflichen Führer fand. [...] die heldenhafte Verteidigung Deutsch-Ostafrikas, die an den sagenhaften Taten einer

140 Man vgl. dazu auch die Personenartikel zu Carl Peters (Bruns 2001: 239-240) und Adolf Lüderitz (Gründer 1987: 452-453) in der Neuen Deutschen Biographie (NDB).

141 „Die Schlacht bei Tanga war wohl die glänzendste Leistung in der Kolonialgeschichte aller Völker und Zeiten, und die in deutschem Soldatengeiste erzogenen Askaris zeigten sich hier ihrer Führer würdig. Sie konnten wahrhaftig stolz sein auf ihren Sieg, und sie waren es auch“ (Mader 1938: 26). „Die Schlacht bei Tanga war von den weittragendsten Folgen: das war nicht nur ein Sieg, der die feindliche Übermacht vorläufig an der Eroberung Deutsch-Ostafrikas hinderte, er gab auch durch seine reiche Beute den von der heimischen Zufuhr und Unterstützung abgeschnittenen Truppen auf Monate hinaus die Mittel zur weiteren Kriegsführung“ (Mader 1938: 28-29). 
grauen Vorzeit erinnert, darf und soll nicht im deutschen Volke vergessen werden! Die Erinnerung an das, was diese Männer geleistet haben, sollte dazu dienen, den kolonialen Gedanken lebendig zu halten. (Darmstädter 1920: 161-162) ${ }^{142}$

Auch der einzige ortsübergreifend erhobene appellativische MoD-Type Askari ist in den zeitgenössischen Kontext dieses in nachkolonialer Zeit publizistisch propagierten Kolonialkriegs zu verorten, der darüber hinaus in zeitgenössischen Benennungsmotiviken verhandelt wurde:

Askaripfad. Im Kampf bei Tanga im Nov. 1914 zeigte sich die hervorrag. Tapferkeit unserer schwarzen deutschen Askaris, der eingebornen Soldaten der deutsch-ostafrik. Schutztruppe, in hellstem Lichte. (Adressbuch München 1941 IV: 78)

In einem unmittelbaren Zusammenhang sind auch die allesamt erst nach 1933 in 30 Groß- und Mittelstädten verfügten SN-Token zur Ehrung und/oder Würdigung Paul von Lettow-Vorbecks zu verorten, der mit Kriegsbeginn zum Kommandeur der „Schutztruppe für Deutsch-Ostafrika“ (Schnee 1914 III: 321) ernannt wurde und im Nationalsozialismus als „zentrale Identifikationsfigur der deutschen Kolonialbewegung - für den ,letzten Kampf um den Kolonialbesitz “ “ (Schulte-Varendorff 2006: 107) propagiert wurde.

\subsection{Ergebnisse}

Für das erstellte Inventar kolonial motivierter Namenvergabepraktiken konnten deanthroponymische und detoponymische Benennungen als die zwei quantitativ dominanten Konstruktionsmuster aufgezeigt werden: Etwa $95 \%$ aller Konstruktionen des kolonialen Namenprojekts in der deutschen Metropole referieren in kommemorativer Intention auf Personen oder auf den kolonisierten Raum. Der Befund, dass vorrangig die Parameter Personalität und Lokalität im (Alltags-)Raum des Deutschen Reichs mittels entsprechender Namenvergabepraktiken fixiert werden sollten, wurde hinsichtlich der damit versprachlichten Wissenskonzepte näher untersucht. Um herauszufinden, welche kolonisatorischen Gewissheiten von administrativer Seite mit derartigen ortsübergreifenden Namenvergabepraktiken tradiert werden sollten, erfolgte eine Analyse der ortsübergreifend verfügten anthroponymischen und toponymischen (mit den jeweiligen Unterklassen) MOD-Types in Bezug auf deren zeitgenössische kolonialdiskursive Funktionen. Die Modifikator-Konstruktionen wurden in enzyklopä-

142 Teil der DSDK. 
dischen Werken und weiteren wissensvermittelnden Texten hinsichtlich zeitgenössischer Zuschreibungen untersucht. Darüber hinaus wurden die in historischen Adressbüchern verfügbaren Benennungsmotiviken hinzugezogen, in denen kollektive Wissensbestände für die Nutzerinnen und Nutzer jener vielfältig rezipierten Quellengattung dargelegt werden.

Folgende Ergebnisse sind dabei herauszustellen: Mittels deanthroponymischer Konstruktionsmuster fand eine Ehrung und/oder Würdigung von Kolonialakteuren des Deutschen Kaiserreichs statt, denen eine wegbereitende Rolle als [Kolonial]pioniere zugeschrieben wurde: Die Personen hatten entscheidenden Anteil am Kolonialerwerb, standen als Kolonialbeamte an der Spitze der zu verwaltenden kolonisierten Gebiete oder hatten die militärische Leitung zur Verteidigung der deutschen Kolonialherrschaft gegen die indigene Bevölkerung sowie gegen die Entente-Mächte im Ersten Weltkrieg inne. Ziel derartiger ortsübergreifender Benennungspraktiken bestand also darin, die Erwerbung, Instandhaltung und militärische Verteidigung der de facto-Kolonialherrschaft durch entsprechende Konstruktionen im Raum der Metropole zu personifizieren. Der deanthroponymische Teilbestand verdichtet sich zahlenmäßig auf die hochfrequente Verfügung der MOD-Types Peters, Lüderitz (jeweils über 60 SNToken), Wissmann, Nachtigal und Lettow-Vorbeck (30 und mehr SN-Token). In diachroner Perspektive ist zu konstatieren, dass sich die Distributionen der zu ehrenden und/oder würdigenden Kolonialakteure hinsichtlich der drei gesellschaftspolitischen Untersuchungszeiträume veränderte: Für den Zeitraum der faktischen Kolonialherrschaft und der Zwischenkriegszeit steht das deanthroponymische Konstruktionsmuster mit dem MOD-Type Wissmann, das auf den Reichskommissar und Gouverneur von Deutsch-Ostafrika Herrmann von Wissmann referiert, zahlenmäßig an der Spitze (Tab. 8 und Tab. 9, Kap. 6.3.2). Dagegen spielt der für das Gesamtinventar dargelegte hochfrequente MoD-Type Peters für Benennungspraktiken bis 1919 und 1933 eine völlig untergeordnete Rolle. Die große Anzahl an über 60 Konstruktionen, die den 1896 seines Amtes enthobenen Reichskommissar Deutsch-Ostafrikas durch ortsübergreifende Benennungen zwischen 1933 und 1945 ehren und/oder würdigen sollten, kann offenbar damit erklärt werden, dass „,in der NS-Zeit [...] der Peters-Kult einen beträchtlichen Aufschwung [nahm]“ (Speitkamp 2004), der auch mittels entsprechender Straßenvergabepraktiken in den öffentlichen Raum fixiert werden sollte. Auch für Paul von Lettow-Vorbeck, der seit Ende des Ersten Weltkriegs „zu den Protagonisten und zugleich Symbolfiguren des deutschen Kolonialrevisionismus“ (Gründer 1985: 358-359) gehörte, ist eine vergleichbare Heroisierung erst nach 1933 festzustellen. Alle 30 Namenvergaben mit dem MODType Lettow-Vorbeck sind ausschließlich für die Zeit nach der national- 
sozialistischen Machtübernahme festzustellen und zeigt, „dass der Kult um die Kolonialpioniere zum einen der Rechtfertigung der deutschen Expansion und namentlich der Vorgehensweise der Eroberer und zum anderen der Schuldzuweisung an die Kritiker einer rücksichtslosen Herrschaftspraxis diente“ (Speitkamp 2005b: 168).

Anhand der historischen Kontextualisierung der ortsübergreifend verfügten detoponymischen Konstruktionsmuster konnte nachgewiesen werden, dass auch mit jener zweitgrößten onymischen Klasse die Fixierung kolonisatorischer Machtansprüche intendiert war. Die mit dechoronymischen und deoikonymischen Konstruktionen versprachlichten Gewissheiten sind weniger eine „euphemistische Verklärung der Fremde“ (Warnke 2009: 49). Vielmehr referieren sie auf die Kolonialgebiete und auf die von der Kolonialmacht als administrative und/oder handelsspezifische Zentren errichteten oder ausgebauten Hauptorte innerhalb der Kolonien. Unter Einsatz von entsprechenden enzyklopädischen Werken und weiteren wissensvermittelnden Texten sind für die usuellen MODTypes zeitgenössische Zuschreibungen festzustellen, die verdeutlichen, dass der Fokus der Kommemoration gerade nicht darin lag, den kolonisierten Raum in Übersee als koloniale Fremde abzubilden. Stattdessen sollten die Kolonien etwas Dazugehörendes sein, die im (Alltags-)Raum des Deutschen Reichs ortsübergreifend als kollektive Wissensbestände fixiert wurden. Dieser Befund ist auch deshalb so herauszustellen, weil toponymische MOD-Types mit usuellen Klassifikatoren für drei und mehr als drei Städte erst nach der faktischen Kolonialherrschaft, vorrangig in nationalsozialistischer Zeit, Verwendung fanden: Nur für Berlin sind bereits bis 1919 koloniale Namenverfügungen festzustellen, deren dechoronymische (Guineastraße, Kameruner Straße, Samoastraße, Togostraße u. dgl.) und deoikonymische (Swakopmunder Straße, Windhuker Straße u. dgl.) Konstruktionen sich auf den faktischen Kolonialbesitz und die dortigen städtischen Zentren beziehen. Die mit praxonymischen Anteilen verfügten toponymischen Modifikator-Konstruktionen verweisen auf die aus zeitgenössischer deutscher Position erfolgreichen Kampfhandlungen und zeigen die überlegene Positionierung der Kolonisatoren in geradezu mustergültiger Ausprägung auf. Die Herausstellung des Eigenen in Übersee konnte auch durch die aufgezeigten Distributionen der mit toponymischen MOD-Types vorgenommenen georeferenzierenden Bezügen dargelegt werden: Der Hauptanteil an ortsübergreifend verfügten Modifikator-Konstruktionen sind der Kolonie Deutsch-Südwestafrika zuzuordnen, die zeitgenössisch die dominante Siedlerkolonie des Kaiserreichs und insbesondere seit Kriegsbeginn das Zentrum der Kupferproduktion für die Metropole darstellte. Die Themen entsprechen damit einer zeitgenössischen kolonisatorischen und eurozentrischen Grundhaltung, mit der die 
wirtschaftliche Nutzung der Kolonien und deren Verwaltung - oder schärfer formuliert: Ausbeutung und Beherrschung - als kollektive Gewissheiten im Raum der deutschen Metropole fixiert wurde.

Anhand zeitgenössischer Quellen konnte dargelegt werden, dass sich die Kolonialbefürworter nicht erst im Zuge der nationalsozialistischen Gleichschaltung, sondern bereits zur faktischen Kolonialzeit und auch in den 1920er Jahren für Fixierungspraktiken kolonialer Kommemoration im städtischen (Alltags-)Raum der deutschen Metropole einsetzten. Für die Kolonialbewegung in der NS-Zeit sind ortsübergreifende Forderungen kolonial motivierter Straßenvergaben festzustellen. Der diachrone Vergleich der von Seiten der Kolonialbefürwortern vorgeschlagenen Konstruktionen und der tatsächlich verfügten Namenmuster durch die städtischen Administrationen zeigt, dass der DKB und der RKB das Thema in den jeweiligen drei gesellschaftspolitischen Untersuchungszeiträumen zum Teil unterschiedlich konturieren, was sich sodann auch auf die Distributionen der ortsübergreifend verfügten MOD-Types ausgewirkt hat (Kap. 6.3.2). Das zeigt umgekehrt auch, dass der Kolonialbewegung einen entscheidenden Einfluss auf kolonial motivierte Namenvergabepraktiken zuzuschreiben ist. 